Scientific Visualization, 2021, volume 13, number 2, pages 117 - 148, DOI: 10.26583/sv.13.2.09

\title{
Modeling and Visualization of Multi-material Volumes
}

\author{
P.-A. Fayolle1,A, L. McLoughlin²,B, M. Sanchez3,C, G. Pasko4,D, A. Pasko5,E \\ A University of Aizu, Japan \\ B Bournemouth University, UK \\ C Uform, USA \\ D NRNU MEPhI, Russia \\ E Skolkovo Institute of Science and Technology, Russia \\ ${ }^{1}$ ORCID: 00oo-0003-4723-6208, fayolle@u-aizu.ac.jp \\ 2 ORCID: oooo-0002-7566-3568, lmcloughlin@bournemouth.ac.uk \\ 3 ORCID: 0000-0003-0501-0112, mathiew@uform.co \\ 4 ORCID: 000o-0001-9688-1698, bstt@yandex.ru \\ 5 ORCID: 0000-0002-4785-7066, a.pasko@skoltech.ru
}

\begin{abstract}
The terminology of multi-material volumes is discussed. The classification of the multimaterial volumes is given from the spatial partitions, spatial domain for material distribution, types of involved scalar fields and types of models for material distribution and composition of several materials points of view. In addition to the technical challenges of multi-material volume representations, a range of key challenges are considered before such representations can be adopted as mainstream practice.

Keywords: multi-material volumes, modeling, rendering, visualization, visual analysis, fabrication.
\end{abstract}

\section{Introduction}

Recently close attention in the research community is paid to heterogeneous volume modeling, which is reflected in several specialized surveys, journal issues and books [MRP95], [MKR*99], [Poo], [Joo], [PSo5], [KTo7], [PACo8], [STG11], [HW13], [PKM13], [RRSS16], [ZJRN18]. This attention is facilitated by growing application areas and a rapid progress in fabrication, in particular in additive manufacturing.

To avoid misinterpretations, we need first to define the terms we use in this survey. Volume modeling is concerned with computer representation of object surface geometry as well as its interior. Solid modeling (homogeneous volume modeling) [H98] deals with volume interior uniformly filled by a single material and with the main question of the point membership classification in relation to objects boundary, interior and exterior. The major characteristics of heterogeneous volume objects are internal spatial variations of single or multiple materials within the volume.

Material is considered a composition of atoms of several chemical elements within the given volume. These atoms can be arranged in some structures such as molecules, particles, grains, crystals and others forming microstructures on several scale levels. A specific material can be characterized by its concentration (or density) representing the fraction of the number of atoms or molecules of a certain type within the taken volume [SBA*16]. Thus, spatial distribution of the material can be represented by a real-valued scalar density field.

The internal spatial variations of materials can occur on the nano-scale level in the form of non-uniform material density distribution throughout the volume (compositional heterogeneity) and on the micro-scale and meso-scale levels in the form of variable spatial structures such as porous structures, lattices, scaffolds and others (structural heterogeneity) [KTo7]. In the case where we deal with some combination of the above types of heterogenei- 
ties, it can be referred to as multi-scale heterogeneity that occurs, for example, on the structural level in the form of nested microstructures [HW13]. Note that in the area of computational material engineering microstructures are considered the carrier of material properties and the variable density distribution is considered a material property derived from the features of nano- and micro-scale structures [S16]. This property is also well understood and considered by the modeling and design research community [MRP95], [RRSS16].

While composite materials can be considered as combinations of multiple homogeneous materials sharing boundaries within a single object, functionally graded materials (FGM) [NSN*87] are composites with two or more material components characterized by continuous gradual changes in material distribution between the components [STG11]. Models of composite materials and FGMs are used in aerospace and other industries, geological modeling, biological modeling, medical simulations, computer animation and visual effects, additive manufacturing and bio-printing. Additive manufacturing makes it possible to digitally fabricate multi-material heterogeneous volume objects and hence becoming the main driving force requiring the development of special design methods and supporting software tools [GZR* 15 , ZJRN18]. For example, the emerging multi-material 3D printers can produce gradual variations between two or three given basic materials by "dithering" them at the highest resolution level [OC14] or by fusing them together at the given volume element (voxel) [HP14]. When these materials represent basic colors such as cyan, yellow and magenta, a full palette of colors can be reproduced.

There are typically three major elements in multi-material heterogeneous object models [KBDH99, PASSO1, ZJRN18]:

1) Overall object geometry. It can be represented by boundary surfaces or by any other solid representational scheme [H98] including procedurally defined scalar fields [PASSo1].

2) Object components (domains/partitions/cells). The entire object can be split into disjoint or adjacent components sharing their boundaries. The space partitions can be defined by additional boundary surfaces [KD97] or scalar fields, which are not necessarily continuous [PASS01]. In the most general case, these partitions are represented by mixeddimensional cells combined into a cell complex [AKK $\left.{ }^{*} \mathrm{O} 2\right]$.

3) Material distributions. Each introduced object component can be assigned an integer value of a material index [KD97] thus providing a model of a composite material. In the case of FGMs, material distribution within a component can be characterized by a single or several real numbers at the given point representing densities or volume fractions of participating materials [KBDH99]. In other words, a single material distribution can be described by at least piece-wise continuous scalar field in contrast to discrete fields represented by scattered sample points or voxel data (see details below).

In general, modeling, optimization, simulation and analysis of material properties at the appropriate resolution can require vast computational resources. This suggests that such properties have to be defined "implicitly" rather than by explicit data structures (voxels, octrees, point clouds) and evaluated on demand [RRSS16]. Continuous scalar fields provide such a procedural approach to modeling with the benefit of resolution independence or practically infinite resolution defined by the computer precision. Such fields also allow for modeling at multiple scales and define infinitely small details. Another advantage of scalar fields is flexible parameterization allowing for interactive redefinitions. Moreover, using scalar fields provides a uniform approach to modeling geometry, FGMs, multiple material blends and microstructures with given material properties directly available for optimization [CSo8] and quick meshfree simulation [FST06]. From the fabrication point of view, scalar fields are suitable for per-point control of the multi-material deposition in 3D printing [DZKo6], [DMO15].

Our main focus is on the ways of representing material distributions with continuous scalar fields within given volumetric domains. The area of multi-material heterogeneous volume modeling with continuous scalar fields has a relatively long history (see [MS95], [JLP*99], [PASSo1], [STO2a], [BSTo4] and other works in this survey). Currently, two extreme cases 
are common in the research literature; it is either an object with simple geometry (e.g., cube or cylinder) with a complex material distribution or a complex object with simple material composition. However, the ultimate goal in this research direction is to provide methods and tools of defining complex, shape conforming material distributions for objects with complex geometry. We mainly concentrate in this survey on modeling material distributions, but the majority of the presented approaches can be applied to modeling internal structures with variable parameters of these structures defined as material properties [FVP13].

We describe different approaches by their representational capacities, intuitiveness, exactness, compactness, efficiency, ease of use, speed, memory, control, connection to simulation, level of complexity, number of materials, robustness of algorithms for discretization (ripping for print) and other characteristics to compare them.

\section{Classifications and characteristics of approaches}

In this section, we present the existing approaches by grouping them according to several classification criteria such as usage of spatial partitions, spatial domain for material distribution, types of involved scalar fields and types of models for material distribution and composition of several materials, also trying to maintain the chronological sequence of publications where it is possible.

\subsection{Spatial partitions}

Using a piece-wise continuous scalar field can be sufficient for describing material distribution within the given object geometry. However, a more intuitive approach is to divide an object into several sub-objects (space partitions) and specify material distribution within each of them.

In classical solid modeling [H98] a solid is considered as a single partition with a manifold boundary with a single material assigned to the entire solid. This approach is supported by modern CAD systems. Later introduction of space partitioning in the form of cellular structures [RO90], [ABC*00] served for supporting mixed-dimensional modeling and nonmanifold and incomplete boundaries.

One of the simplest ways to define a heterogeneous object is to introduce space partitions sharing their boundaries and splitting the object into several sub-objects. Then, each space partition is assigned a single uniform material. The work [KD97] introduced a multi-material heterogeneous solid as a collection of interior disjoint regions each filled by a single material represented by its integer index. All the regions have manifold boundaries and can have common boundary elements. A corresponding multi-material extension of the geometric file format for additive manufacturing STL was proposed in [CTOO]. A similar approach was developed in [YYW12] with boundaries of multiple partitions represented in the piecewise polynomial form based on an adaptively subdivided octree and a single material assigned to each partition. An application of such an approach to heterogeneous fruit modeling was presented in [BTG15] on the basis of 3D geometric maps and L-systems.

Voxel arrays in volume modeling can be considered a simple example of spatial partitioning with a single material assigned to each regular hexahedral cell (voxel). Voxels are currently considered as a model suitable for multi-material 3D printing [HLo9c], [DTD*15], [M15]. However, this model is an essential approximation for the object geometry and material distribution due to the fixed resolution. Growing the voxel resolution eventually causes serious problems with both the data structure storage memory and its processing time.

The model of [KD97] is sufficient for representing composite materials, but not for modeling FGMs. This was amended in [KD98] by introducing a vector of volume fractions of materials participating in the composition. The material composition is thus represented by a vector material function of volume fractions that must sum to unity at any given point. A cor- 
responding CAD system architecture was presented in [ZCFO5], which also included microstructure design along with FGMs.

Works [JPSC98, JLP*99] proposed subdividing the object into specific sub-regions (called finite elements). Tetrahedral finite elements were discussed in detail with the possibility of extending to other types such as hexahedral, wedge, and pyramid. To represent material distributions, an analytic blending function is assigned for each material using Bernstein polynomials of barycentric coordinates within each finite element.

In [KBDH99] the heterogeneous solid model of [KD97, KD98] was generalized in the $o b-$ ject model by representing geometry as a topological cell complex (disjoint composition of closed 3 -cells) with pointwise object attributes represented by a collection of $\mathrm{C}^{\mathrm{k}}$ continuous functions (scalar fields). Each attribute represents some object property at the given point such as density or volume fraction of participating material. Thus FGM can be modeled separately for each spatial partition. The work [BSDoo] extends this theoretical approach to practice by listing several analytical material composition functions suitable in various FGM applications and by outlining the design process of heterogeneous objects.

In [PASSo1] the constructive hypervolume model was introduced for representing heterogeneous objects as general multidimensional point sets with pointwise attributes. The Function Representation (FRep) [PASS95] is used to represent both geometry and attributes by scalar fields. The main distinctive feature of FRep is that the function is procedurally evaluated using an n-ary tree structure with primitives in the leaves and operations in the nodes. Such typical operations are set-theoretic ones defined with R-functions [So7]. The constructive hypervolume model is represented by a vector function with one component (continuous scalar field) defining geometry and other components defining attributes. All the function components have associated tree structures. Each function is procedurally evaluated by traversing the corresponding tree structure. Attribute functions can be discontinuous and are based on FRep of space partitions.

The authors of [CDM*02] presented a procedural approach to authoring layered solid models, which is a volumetric representation with nested spatial partitions called layers. An object is bounded by one or more closed polygonal meshes. Each layer is defined by a range of values of the signed distance field (SDF) to the object boundary (or some function of this field) and has a material type assigned to it. Color and density of each material are defined procedurally and can vary within the layer. A volumetric object is similarly partitioned in [WYZG11] by SDFs to object regions. A heterogeneous object is similarly described in [LFAB14] using nested polygonal meshes bounding the regions with different material density values. The work [SK16] uses binary space partitioning (BSP) to model multi-material objects. The standard BSP with separating planes is extended by curved surfaces separating regions with different materials. FGMs are modeled using SDF of the region boundary. The separating isosurfaces for the BSP model can be extracted from voxel scanned data. Similarly [GT15] employs specific primitives to bound space partitions for FGMs within the given object geometry. They universally employ for these purposes convolution surfaces with point, straight line, spline and plane skeletons.

The Foundry system [VKWM16] allows the user to interactively decompose the initial volume object into multiple sub-volumes with the graph-based interface and the underlying procedural definitions. Several modes are supported for partitioning the object such as operators based on distances from surfaces, curves and points, partitions based on geometric primitives with the known point membership classification, and discrete uniform lattice decompositions.

In $\left[\mathrm{AKK}^{*} \mathrm{O} 2\right]$ the authors introduced a hybrid cellular-functional model based on the notion of an Implicit Complex (IC) that was defined on the basis of CW complexes. It provides a valid topological description of heterogeneous objects and allows for a flexible combination of cellular and function representations of both the geometry of objects and their attributes. Geometry and attributes are defined independently and occupy their own part of multidimen- 
sional modeling space. Thus a heterogeneous object is represented by a union of high-level components (cells) that can overlap each other. A number of the special constraints on the description of the mutual dispositions of these components were introduced. That effectively results in a cellular topological subdivision. The intersections of the components are described by constructive methods which preserve the precision of the representation. The model is inherently multidimensional: the object can include components of various dimensionalities. It combines the advantages of both the topological and constructive representations. ICs have found an application in modeling highly complex geological heterogeneous structures in the petroleum exploration [BKK $\left.{ }^{*} 15\right]$.

Smooth transitions between different porous structures are considered in [YQZT14]. For two given porous materials, a boundary surface between two space partitions is defined implicitly and a special weighting sigmoid function is introduced to define the transition. Then, this approach is extended to multiple porous materials each defined within an assigned space partition.

The work [FSP15] employs specific spatial partitions based on a Voronoi diagram built for interior distance fields defined by material feature points introduced by the user. Transition zones to interpolate between materials are created by intersecting offsets of adjacent Voronoi cells. The interpolation between material properties within the transition zone is implemented using a form of the transfinite interpolation [RSSTo1] (for details, see 2.4).

An alternative to using spatial partitions is a fully heterogeneous mixture of gradually changing multiple materials within the entire object volume. This can be achieved in different ways such as source-based or fully procedural modeling as described in 2.4 .

\subsection{Spatial domains for material distribution}

Material distribution can be described in various ways in respect to the spatial domain dimensionality (from one-dimensional to space-time and multidimensional domains) and gradient directions.

The gradient is the vector defining the direction of the function variation at the given point. If the material property such as density is changing only in a single direction, [HMM11] calls it a one-dimensional (1D) gradient. It means there is some constant vector parallel to the gradient at any point in space. Several examples of $1 \mathrm{D}$ distributions defined by continuous functions of a single coordinate are given in [MRP95]. Another example is a radial material distribution specified in a cylindrical or spherical coordinate system [BSDoo]. The variations of the property along the chosen direction can be defined analytically, procedurally or using samples from a curve input by the user through a graphical user interface (see 2.4). The gradient is two-dimensional (2D), if there are two constant vectors resulting in their linear combination equal to the gradient at any given point. A three-dimensional (3D) gradient is by analogy a linear combination of three constant vectors. The work [HMM11] provides a full classification of material gradients of various dimensionalities and [KPT12] gives a brief survey of related works.

Some examples of $1 \mathrm{D}$ material distribution can be found in [GT15], where 2D distributions are obtained by combining two or more one-dimensional distributions with assigned weights called dominating factors that have to be normalized to sum to unity. The dominating factors in their turn can be non-linear functions of point coordinates providing more control for 2D material distributions. In [XSo5], a 2D material distribution is generated at each horizontal slice on the basis of the object offset iso-contours. By analogy with the $2 \mathrm{D}$ case, a $3 \mathrm{D}$ material distribution can be obtained as a combination of several $1 \mathrm{D}$ and $2 \mathrm{D}$ distributions with assigned dominating factors [GT15]. A user-guided generation of a $3 \mathrm{D}$ distribution from multiple 2D distributions given for orthogonal object cross-sections is presented in [TSNI10]. A fully volumetric $3 \mathrm{D}$ material properties distribution is called a solid texture in computer graphics [PCOS10]. 
All works on animation or simulation of time-variant volume objects formally deal with 4D space-time domain. Typically, they employ discretization in the form of either finiteelement meshes $\left[\mathrm{CDM}^{*} \mathrm{O} 2\right]$ or voxels in some variation of the level set method [S99]. The work [SFA*15] is one of the first attempts to find analytical solutions in $4 \mathrm{D}$ for shape conforming multi-material transitions purely based on scalar fields following the general multidimensional formulations of [PASSo1].

\subsection{Types of scalar fields}

Numerous methods for heterogeneous volume modeling rely on a scalar field to control the material properties, whether directly or as a part of a material function depending on the distance. Such a field usually provides some measure of the distance to the object boundary or to some geometric feature, or can be represented by other geometry-dependent functions. Several properties of the scalar field must be considered: smoothness, user control and predictability, as well as its computational cost.

Smoothness here means at least $\mathrm{C}^{1}$ continuity of the field function. In [BSTo4], the authors argue that 'the lack of smoothness in a material function will result in stress concentrations and other undesirable effects. Smoothness is not a strict requirement, but can be desirable for a number of applications. User control is necessary for practical users of a modeling system (engineers, designers, artists) to create a material function which describes exactly their intent. The distance field is often necessary to provide predictability as argued in [BSTO4], [FST06] and [FSP15]. In addition, [SD01] argues that the lack of distance properties makes it difficult for the user to control or predict the material distribution. Finally, such a property as computational cost is often overlooked, however this criteria cannot be ignored because the design process requires some level of interactivity and many iterations.

The following subsections cover the types of scalar fields used in heterogeneous volume modeling in several categories. Note that instance based fields, exact or approximate, have been prevalent in the subject area and therefore represent most of the existing literature.

\subsubsection{Exact distance}

In general, a continuous distance field defines the object geometry within the given computational precision [HSS14]. Euclidean distance fields and signed distance fields are also good candidates for constructing material functions. Distance functions can be used to define gradual changes of FGMs with simple geometric primitives such as points, lines and surfaces as field generating features. These features are often easy to define for the users and the distance fields for them are trivial to implement. The generated distance functions are also intuitive for the designers and engineers. The only drawback is the lack of smoothness inherent to exact distances.

The work [JLP*99] divides a model into several cells and then, for each cell, a material function is defined. This function depends on various distance functions to several elements such as vertices, lines or facets. In $\left[\mathrm{CDM}^{*} \mathrm{O} 2\right]$, the authors also propose to use distance functions, but with offering more flexibility through the use of a scripting language. This flexibility limits the type of users, but allows for more precise control of the field. While [CDM*O2] recommends signed distance fields, other fields are also suggested by changing the interface velocity during the signed distance field computation. The works [BSTo4] and [ZLLO9] create discrete distance fields for all the provided features and combine them into a single material function. The paper [GKT12] introduces gradient references, where the user can define two simple geometric elements, and the distance fields are blended together to control the material distribution.

Overall, exact distance functions are popular because they are computationally efficient (see, for example, [SFP12]) and succeed in all but one criterion, which is smoothness. This is the reason of the introduction of approximate distance fields discussed below. 


\subsubsection{Approximate distance}

As we mentioned earlier, exact distance fields are inherently $\mathrm{C}^{1}$ discontinuous, which often leads to a lack of smoothness in the material function. This has led to the introduction of several pseudo-distance fields which attempt to preserve the exact surface, but get smoother elsewhere. In [FPSMo6], a smooth approximation of the min/max operators (used to implement set operators) was introduced in order to create smooth material functions. These operators enable the users to build complex and smooth distance fields for set-theoretic combinations of simple primitives. Several works ([FPA11], [BFP13], [SFFP15]) provided methods to calculate smooth approximate distance function to polygonal meshes. In [FPA11], binary space partitioning and smooth R-functions are used to create a function, which can approximate the distance function to a polygonal mesh. In [BFP13], signed $\mathrm{L}_{\mathrm{p}}$ distances were introduced, which use the mean value normalization function. A convolution filter with varying radius is applied in [SFFP15] to the exact distance function of the mesh. All three methods succeed in producing a smooth approximate distance function, but can be computationally expensive.

\subsubsection{Other scalar fields}

An important issue with distance based methods in heterogeneous object modeling is that typically the shape of the object is not taken into consideration by the material function. A logical step is to use interior distance, which can provide shape conforming material distribution. Numerous approaches exist to compute interior distances, such as [RLFo9] or [CWW13]. In [FSP15], interior distances were used in the combination with the transfinite interpolation [RSSTO1] to achieve shape conforming distribution of multiple materials defined by the user provided material sources.

Another attempt to provide more intuitive control of the material functions is using distances to basic primitives (splines, spheres, etc.) for generating "material potential functions". Here, the basic primitives influence their surrounding regions based on that potential functions [GT15], which maps distance to a "potential". It can represent abrupt changes with a step function, for instance. It is easy to use and complex models can be made pretty quickly. This model offers compactly supported material properties, which means potentially fast processing, but it is limited in the sense that it loses sensitivity to the distance quite quickly.

\subsection{Material distribution models}

The ways scalar fields defining material properties are specified depend on the type of available data and the selected mathematical model. In this section, we consider the main classes of them.

\subsubsection{Sampling and interpolation schemes}

Sometimes material distributions are prescribed directly by the user through scalar or vector valued functions defined and available within the domain. Alternatively the material distributions may be specified on a domain boundary only and then interpolated within the domain. In the other cases, these distributions will be available only as samples, either as nodes of a grid (regular or irregular) or at scattered points, and interpolation will be required for further applications. These samples can be the result of measurements, for example, via photogrammetry, optical scanning, or electromagnetic methods, or the result of some numerical simulation, for example, by solving numerically some partial differential equations.

\subsubsection{Grid interpolation}

Often material samples will be available in the nodes of a grid, either a regular grid or an irregular grid such as a tetrahedral mesh [Poo]. The former may be the result of simulation by the level-set methods [OFo6], while the latter is likely resulting from some simulation by the finite element method [ZT77]. In these cases, the linear interpolation is typically used. 
However for some particular applications, higher order polynomials are sometimes used, such as, for example, quadratic super splines in [RSZNo4] and a non-linear weighting scheme in [KFC* ${ }^{*} 7$ ].

Varying material properties (such as in FGMs) can be created and controlled by spatial variation of material structures. In the work [LS17], Liu and Shapiro propose to represent and control material structures using techniques for texture synthesis. Material structures are modeled with Markov Random Fields. The properties of a target structure are used to guide the structure synthesis from a given discretized reference material structure and its known properties. To simplify the computations, the direct evaluation of the material properties is replaced by the computation of a proxy descriptor. Examples of descriptors include correlation functions or Minkowski functionals. The authors illustrate their approach with the synthesis of the missing part of bone, and with the generation of a FGM structure from a target volume fraction distribution and a reference material.

\subsubsection{Scattered data interpolation}

When data is obtained as a result of measurements, often there is no connectivity available between the samples, which are available at scattered points only. One approach consists in introducing connectivity by computing the Delaunay triangulation (or its dual the Voronoi diagram) of the points to find the natural neighbors of a given evaluation point and then using some interpolation method, the so called natural neighbor interpolation. The Sibson coordinates [S81] fall in this category. Hiyoshi and Sugihara [HS99] extended this approach by using different weights for the interpolation; the corresponding approach is named Laplace interpolation (or Laplace coordinates) as it corresponds to a discrete approximation of the Laplacian. In [HSoo], the authors show that Sibson and Laplace coordinates are parts of a same family of coordinates defined on a power diagram (or Laguerre Voronoi diagram) of the input scattered points. Laplace interpolation is used in computational mechanics, for example in [SMSB01].

Inverse distance interpolation, also called Shepard interpolation [S68], is another technique designed for scattered data interpolation. At a given point of evaluation, each sample contributes with a weight inverse to the distance of the evaluation point. A transfinite version of this interpolation technique was used for defining gradient material from material distribution prescribed on features in [BSTo4] and in [FPSM06]. More recently, it was also used to perform animation of heterogeneous object in [SFA*15]. Inverse distance weighting using natural neighbors was used to interpolate heterogeneous features in [FSP15], with the intrinsic distance (with respect to the solid) to the different features being used in the interpolation.

Another approach for interpolating scattered data is to use interpolation with radial basis functions (abbreviated in RBF) [Boo]. RBF interpolation was used in [TRSo4] to reconstruct a surface with interpolated attributes from a point-cloud with attributes. In [Y13], RBF interpolation was used for representing $3 \mathrm{D}$ heterogeneous objects. In contrary to [TRSo4], the attributes are not limited to the surface of the object but are specified and interpolated within the object.

When the sample points belong to a small number of classes (materials), the problem can be interpreted instead as a classification problem (multiclass classification). The input is a set of labelled sample points, with each point being taken from one of the material classes. Then one needs to train a classifier, such that it can predicts at each point in modeling space the class (material) that this point belongs too. This approach was used in [YYW12], where multicategory Support Vector Machines (SVM) are used to train linear or quadratic polynomials. Since the training on large data-set can be computationally expensive, the input samples are first subdivided in smaller subsets by using an octree. The classifier is then trained in each leaf. The distance to the separating hyper-surfaces are then combined with a blending scheme inspired from [OBATSo3], and used for the final classification in the whole modeling space. 


\subsubsection{Boundary interpolation}

In some cases, the material distributions can be specified on the boundary of some domain and have to be interpolated within the domain. When the boundary is specified by polylines (in 2D) or triangles (in 3D), the material distributions are samples on the element nodes, and interpolated within the domain using generalized barycentric coordinates. The usual types of barycentric coordinates for interpolation include mean value coordinates [Fo3b, FKRo5, JSW05], Voronoi [JLWo7] and Wachspress [W75] types. Interpolation of surface attributes to the domain interior with mean value coordinates is shown as an example in [JSW05].

When the boundary is given by a smooth curve (in 2D) or a smooth surface (in $3 \mathrm{D}$ ), transfinite (continuous) interpolation is used. Early work by Gordon and Wixom introduces methods for interpolation of function value and derivative [GW74]. Mean value coordinates are extended by Dyken and Floater for the transfinite case in [DFo9]. Transfinite versions of the main barycentric coordinates are given by Belyaev, as well as extension of the Gordon-Wixom coordinates to general domain [Bo6]. The weighted generalization of the transfinite mean value can be considered as a transfinite version of the Shepard interpolation [S68]. Curiously, it seems that most of these techniques are not really used for the modeling of heterogeneous material objects, with the exception for the Shepard interpolation, which was used for transfinite interpolation of material prescribed on some features in [BSTo4, FPSMo6, BFSSo8, FSP15].

\subsubsection{Simulation and optimization}

Description of the material distributions is either provided by the user, eventually only on some subsets, or is the result of some measurements (or experiments) or can be even obtained via the result of some numerical simulation. The distributions can also be obtained from an optimization process where the material distributions are modeled to optimize some user specified objective function. The typical tools available for optimization problems include: gradient descent, stochastic optimization, simulated annealing, particle swarm, quadratic programming, conjugate gradient, branch and bound, Newton Raphson, Lagrangian methods [ZCFo4]. When the material distributions are obtained from a numerical simulation, we can use tools such as: finite element methods (the Galerkin approach, etc), meshfree methods (such as Ritz-Kantorovich method), or the level-set methods.

In [HLo9b] heterogeneous materials are represented by voxels. The shape or the material distribution is optimized with an evolutionary algorithm guided by constraints, such as maximizing stiffness per weight or some higher level constraints. There are many works in the literature for shape optimization, both geometry and topology [A12]. An often used approach is based on level-set methods [OFo6]. This was naturally extended to work with multi-material objects. One approach for the optimal design of multi-material objects is to represent them implicitly with multi-phase level-sets. Shape and material optimization can be done via solving partial differential equations using level-set methods [WWo5, CCZ16], or by optimizing some variational criteria [WLKZ15].

Optimizing the design of FGMs is considered in [HFBGo2] by optimizing two objective functions that depend on the volume fractions of the primary materials. In [GVo7] the material composition for two model problems is optimized by an element free Galerkin analysis combined with a genetic algorithm. In [KPT12], generic material heterogeneity is represented by feature tree based procedural models, and the material composition for the functionally graded materials is optimized by the nature inspired Particle Swarm Optimization. Chen and Shapiro [CSo8] proposed to represent heterogeneous objects with parametrized implicit surfaces and Constructive Solid Geometry (CSG) operations (such as union, intersection and subtraction), and to optimize the parameters to some specified constraints with gradient descent. 
Several recent works model stochastic heterogeneous materials with an optimization process. Periodic and stochastic microstructure objects are reconstructed with the Simulated Annealing algorithm in $\left[\mathrm{PFV}^{*} 11\right]$. Liu and Shapiro proposed to reconstruct material models as a Markov Random Field texture synthesis [LS15]. Their method presents several advantages over traditional optimization procedure, such as an improved computational efficiency. Finite element methods are used in $\left[\mathrm{CDM}^{*} \mathrm{O} 2\right]$ for performing some simulations on procedurally defined heterogeneous solid models.

Heterogeneous objects are modeled by a diffusion process in [QDo3]. The diffusion process is solved by finite element methods. Geometry is specified by B-Splines. Additional constraints on the material distribution are considered by solving a linearly constrained quadratic optimization problem with the method of the Lagrange multipliers.

Instead of finite element methods, Tsukanov and Shapiro propose to use meshfree methods for modeling fields in heterogeneous objects [TSo5]. Materials are either explicitly defined or prescribed on material features and interpolated within domains using inverse distance weighting [BSTo4]. A meshfree method is used to solve problems such as heat diffusion. A similar approach is used in [FSTo6] for more complex geometrical domains, to which the distance is sampled and interpolated with B-Splines.

\subsubsection{Analytical (closed form) models}

In the case of a relatively simple and well-known type of the material distribution, it can be described in the closed form of an analytical expression over the point coordinate(s). Such expressions. These expressions can be derived by researchers via empirical analysis of experimental data or through some automatized derivation and parameters fitting.

Early studies of FGM modeling such as [MRP95] discussed volume fraction distributions for materials in the form of polynomials of degree $\mathrm{N}$ varying from 0.25 to 4 . The coefficients of such a polynomial can be empirically defined by the researcher or automatically optimized under certain constraints [MS95]. This type of distribution is called the power law in [KD98]. Logarithmic material distribution functions are mentioned in [GKT10].

Thee work [BSDoo] presents a variety of analytical material composition functions including polynomial, exponential and trigonometric ones and proposes to have a library of such functions available in a design system to satisfy requirements of different application areas. From the FGM manufacturing strategies point of view, [HMM11] provides examples of material functions for a mechanical part (with several domains) including trigonometric functions and polynomials. Analytical functions can be used not only for defining individual material distributions, but for specifying smooth transitions between constant material properties with the given boundary [YQZT14].

File formats for additive manufacturing and computational material engineering have adopted the closed form functions for the material distribution. Thus, the relatively new file format for additive manufacturing called AMF supports defining FGMs by introducing analytical functions of point coordinates and limited procedures for each material distribution in space [HLO9a, ASTM11]. The STandard for Exchange of Product data (STEP) has capabilities for specifying heterogeneous materials and analytically defined FGMs with introduction of new entities conforming with the data structures of STEP [PDB* OO]. In computational material engineering, material properties such as concentration of atoms of a certain type are represented in the $\mathrm{HDF}_{5}$ file format [SBA*16] as continuous scalar fields using predefined mathematical functions and operations.

Kou et al. [KPT12] give a number of examples of analytically defined material distributions and conclude that it is not always simple to derive an analytical function for the entire geometric domain and the function selection can be a subjective choice of the designer based on their experience. With using too simple analytical models for material distribution, inconsistency may appear in the material property representation due to approximation errors 
[STG11]. In this sense, sample-based models provide more objective and unbiased material distribution models.

\subsubsection{Spline-based models}

In the domain of heterogeneous object modeling, splines can be used as an interpolation tool, for interpolating values obtained from measurements or from a numerical simulation, or they can be used directly in the modeling process. The latter is seen in [JLP*99, JPSC98], where heterogeneous objects are modeled by subdividing a solid object into cells, and associating blending functions to each cell. More specifically, shape and material composition of each cell is formulated in terms of control points blended by Bernstein polynomials. Splines, such as Bezier splines, B-Splines or NURBS, can also be used for representing domains parametrically (as in the previous work) or implicitly. Schmitt et al. [SPSo4] use B-Splines both to define implicitly solid objects with their spatial partitions corresponding to the different material and attribute (material) distributions.

In [HQ05], the authors use tri-variate simplex splines to represent both the object geometry and the object attributes. Due to the flexibility of simplex splines, this approach can be extended to support multiresolution modeling of heterogeneous objects. It is also described how to fit tri-variate simplex splines to acquired heterogeneous data.

Instead of simplex splines, Yang and Qian [YQ07] use B-Splines to represent both the geometry and the material composition of heterogeneous objects. They introduce the concept of heterogeneous lofting, where the lofting method for free-form surface modeling is adapted for modeling heterogeneous objects. Given a set of material profile features, a heterogeneous object passing through both the geometry and the material compositions is constructed.

Similar techniques are used in the domain of medical modeling: a B-Spline solid representation is extended in [WBo9] for representing material composition in order to develop a heterogeneous model of the human body. The model is created slice by slice from CT scan data, either by surface fairing or by surface fitting. Similar techniques are further improved by Grove et al. in [GRP12].

A technique based on gluing heterogeneous cells modeled by splines is considered in [QD04], [CTGF15] and [ME16]. More precisely, Conde-Rodriguez et al. [CTGF15] model heterogeneous objects as tuples: $\mathrm{O}=\{\mathrm{S}, \mathrm{V}, \mathrm{F}\}$, where $\mathrm{S}$ is a r-set corresponding to the geometry, $\mathrm{V}$ is the set of all valid material distributions, and $\mathrm{F}$ is the material function. The material function is defined by considering its restriction on a partition of $\mathrm{S}$ into cells. Each cell is represented by a Bezier hyperpatch, where cubic Bezier functions are used to blend control points corresponding to the geometry and the attributes. Massarwi and Elber [ME16] instead consider B-spline trivariates for modeling volumetric cells similarly to [QDo4]. A V-model is then obtained as a complex of several such cells. Additional operations on V-models such as Boolean operations or material composition are also defined within their heterogeneous modeling framework, however these are not directly represented by B-spline trivariates.

The majority of spline-based approaches for heterogeneous modeling are built on Bezier splines, B-splines or related. The use of RBFs such as thin-plane splines is however much less common. Tobor et al. used RBF combined with a partition of unity to fit heterogeneous implicit surface to point-cloud with attributes [TRSo4]. The approach is limited to surface attributes though. A similar approach was proposed by Yoo in [Y13], where RBF are used to interpolate control points with attribute values, and a heterogeneous object is then defined from the solid with interpolated material distributions.

Spline-based models are suitable for internal representation in some application areas. However, they are not always convenient from a user interface point of view, because the definition of volumetric material distribution via the manipulation of control points is not very intuitive. In this case, other models (such as source based model described below) can be used for the user interface and then converted to a spline-based model. It is also possible to use splines to fit material distribution obtained from measurement or physical simulation. 
Another restriction of spline based models is that they are not closed under set-theoretic operations and need some additional data structures to support such operations.

\subsubsection{Source-based and feature-based models}

As it is stated in [LMP*04], methods based on volumetric meshing or cellular decomposition do not support simultaneous editing of geometric and material models and introduce approximation errors at early stages of design. Spline-based methods are not intuitive for designers although can serve well for internal material representations.

A source- (or feature-) based description of material distribution is a practical alternative to analytical functions and discrete samples interpolation, and complementary to splinebased methods. It allows the designer to identify some high-level geometric elements or parts of the shape and to assign a material distribution function (homogeneous material in particular) to each of them for creating sources of grading materials [JPSC98, QD98, PCBoo, STo2a, BSTo4, QD04]. It is supposed that materials are blended in the rest of volume, for example, with weights proportional to some distance measure for each of the selected elements. Material fractions are interpolated for the points inside the volume according to these weights.

Early works on feature-based FGM representations [JPSC98, PCBoo, PCo3] proposed to assign some material distribution functions to elements of object boundary (vertices, edges or faces) and then to blend them for the interior points of the volume using inverse distance weighting. The work [LMP* 04 ] proposes to numerically solve Laplace's equation to compute material blending from the boundary conditions derived from the material compositions assigned to surface or volume features. A lofting operation is used in [SKo4] to blend between lower-dimensional material features, which can be automatically detected through an optimization process based on object's functional requirements and constraints [SKo5, SKo8].

In [JPSC98, STo2a, STo2b], grading material sources (fixed references, origins of material variation) can be points, lines and planes arbitrarily placed in space as well as outer object boundary surface. Each grading source is associated with three partitions of the heterogeneous object: an effective grading region, where the material composition function is changing from $\mathrm{o}$ to 1 , and two complimentary regions, where this function is constant, $\mathrm{O}$ and 1 respectively. The material composition at the given point within the effective region is evaluated according to the distance of this point from each source. Volume fractions of all materials at any point have to form a partition of unity. The work [GWB ${ }^{*} 12$ ] uses $3 \mathrm{D}$ polylines or spline curves as material sources and skeletal-based functions defined in the local coordinates for modeling continuous material properties such as porosity or permeability in geological modeling.

This method has found its further generalization in [BSTo4], where sources are replaced by material features each represented by its own scalar field (continuous function) and having prescribed material properties, and the transfinite interpolation [RSSTo1] used to obtain the material distribution at the given point in space based on the functions defining material features or their normalizations with distance properties. The work [Poo] mentions practical difficulties of using such an approach because of the computationally expensive conversion of parametric surface boundaries to implicit surfaces defined by scalar fields and conversion of complex boundary models to constructive models with R-functions [So7]. A discrete version of the feature-based model for a regular grid with applications in additive manufacturing was presented in [ZLLO9]. The step-like transition functions between space partitions [YQZT14] can be considered an extension to the case where there is no gap between neighbouring material features sharing a common boundary. This approach is especially suitable for simulating physical fields within heterogeneous objects [TSo5] and to extend product development process specifications [BFSSo8]. Blending between several material features was further developed in [OK11] with imposed relations and constraints on them. Offset curves of feature boundaries and their optimized metamorphosis are used to define the material composition between several materials maintaining the relations and satisfying the constraints. In 
[TOIIo8] the authors propose an interactive method for placing exemplar $3 \mathrm{D}$ texture patches according to a user-defined volumetric tensor field.

Using Euclidean distance to interpolate between material sources or features not always provides intuitive results, especially in the case of quite complex shapes. To take the object shape into account, Euclidean distances can be replaced by interior distance measures evaluated as the minimal length of the path between any two object points, which completely belongs to the interior or the surface of the object [FSP15]. In general, the interior distances can be expressed in a continuous setting, however, in practical applications usually approximations such as regular grid sampling are used.

\subsubsection{Compound/constructive models}

An alternative and a complement to the feature-based modeling is a constructive approach to building "compound" models starting from simple primitives (partitions, regions or building blocks) with known material distributions and then combining them with analogues of set-theoretic, blending or other operations applicable to heterogeneous object models. Such a model is characterized by a user-defined or automatically established hierarchy of operations and/or topological relations between different object partitions and material features.

First, we present several specific hierarchical structures developed for heterogeneous objects and serving various purposes.

The system Svlis [B95] was based on an advanced CSG system extended by homogeneous material properties assigned to each primitive and then evaluated for each node of the construction tree. A similar approach was proposed in [KD97] as a mathematical constructive model for solids composed of multiple homogeneous materials. A $3 \mathrm{D}$ solid is subdivided into partitions assigned with unique materials. A non-manifold Boundary Representation (BRep) scheme is used to represent such objects. Each partition is homogeneous inside and has an index of material assigned to it. Regularized set-theoretic operations are applied to the solid components as point sets. Corresponding operations on material indices are introduced on the basis of selection by the user of the resulting material for each pair of materials and for each set-theoretic operation.

In spite of the ways homogeneous material regions have been constructed, [CToo] proposes to represent the entire multi-material object hierarchically with a "material tree" of possibly nested boundary surfaces of homogeneous materials. Addition or removal of a homogeneous material region in the object results in rebuilding the tree structure. If the given point belongs to multiple regions, the priority is given to the material index of the region lowest in the tree structure.

Brochu and Schmidt propose in [BS17] to use non-manifold triangle meshes to represent multi-material objects. They describe an interactive interface for letting a user create such non-manifold surface meshes by marking existing objects. They also describe a technique for implementing non-regularized Boolean operations that can be used as a general method for the creation of non-manifold surfaces.

The Hierarchical Feature Tree (HFT) [KTo5] structure similarly to BRep hierarchically organizes heterogeneous features with their geometry and material variation dependency relationships. In HFT, k-dimensional heterogeneous features are built from (k-1)-dimensional ones using linear or inverse distance interpolation as well as extrusion and revolution operations. The optimized procedural evaluation of material compositions based on HFT was presented in [KPT12]. While HFT allows for modeling material distribution for a single part, the extended Hierarchical Feature Tree (eHFT) [KTo8] supports several object partitions, each associated with its own HFT, thus forming heterogeneous cells combined within a nonmanifold cellular structure.

A volumetric object is decomposed in [WYZG11] into several partitions called regions with each region represented by several Signed Distance Functions (SDF) to its boundary 
parts. A region is uniquely identified by a set of signs of corresponding SDFs, which are organized in a binary tree structure called an SDF tree. Each leaf of this tree corresponds to a region. A material attribute (color in this case) function of point coordinates is assigned to each region. These can be solid colors, solid textures (discussed below) or RBFs interpolating attribute values given at random points of the region. One object can be embedded into a region of another by linking its tree to a leaf node of the containing region thus creating a multi-scale structure.

The reducer tree [CLD*13] generalizes the material tree and several spatial partitioning data structures, and serves for both specifying object spatial partitions and assigning material distribution to each of them. There are two types of nodes in the reducer tree, namely geometry nodes and material nodes. The entire given object is assigned to the root of the tree. Geometry nodes are internal tree nodes used for specifying spatial partitioning of the object. For example, a B-spline node splits the object into two partitions and serves as a boundary surface between them. Material nodes are leaves of the reducer tree and they assign specific material to the specific spatial partition including FGMs defined by a function of point coordinates.

Some specific operations on material attributes and heterogeneous objects have been developed and can be included in the hierarchical structures presented above. Simple constructive set operations on primitives with assigned different homogeneous materials are described in [ZSo4], where one of the materials can dominate in the result due to the selected type of the union operation in composite material or microstructure design. Geometric operations with associated attribute operations are considered in [KBDH99] for the introduced object model. Operations on attributes are applied in conjunction with operations on geometry (such as regularized set operations) to produce an appropriate corresponding attribute model for the resulting geometry. "Combine" is such a typical operation necessary for generating material composition values when combining two heterogeneous objects with set operations. Such an operation can differ for each attribute depending on the individual attribute model. Vector space operations applicable directly to attributes such as summation and multiplication with a scalar are also introduced. The "combine" operation for the material composition was further elaborated in [SD01] by introducing a material Boolean operator, which takes into account the given point location in respect to the intersection area of two objects and interpolates between two material composition functions using the distance to the object boundaries. Similar modifications were applied to derive set operations on source-based heterogeneous objects in [STo2a]. New types of operations such as immersion, insertion and merge were also introduced allowing for various combinations of geometry and material distributions. A prototype heterogeneous CAD system based on set (Boolean) operations over heterogeneous objects is described in [BSDoo]. Note that at any point within the object the material is represented as a combination of the primary materials of primitives satisfying the partition of unity of volume fractions and allowing for FGM modeling.

Material composition operations have found further extension in blending composition functions associated with set operations on geometric point sets. By analogy to the featurebased modeling discussed earlier, [SD01] applies the transfinite interpolation [RSSTo1] to material composition values, where defining functions of geometric primitives and subsets involved in the set operations serve to evaluate the weight for each material composition.

As it was shown in [BSTo4], blending of material compositions between material features represented by scalar fields can be implemented using the transfinite interpolation [RSSTo1]. Such a blending operation on material compositions was extended to the space-time domain in [SFA*15]. For a time dependent transformation of a heterogeneous volume given by its initial and final states, the proposed space-time blending applies the transfinite interpolation in $4 \mathrm{D}$ space-time to provide material compositions for intermediate states of the volume object. 
Some other types of primitives and operations on heterogeneous objects have been proposed in literature. For a given two-dimensional generator area and a defined path, a swept solid can be generated. With a material composition function defined for the $2 \mathrm{D}$ generator, [SD01] specifies material distribution within the $3 \mathrm{D}$ swept solid using an additional mapping for the given point in space to the material composition value.

To increase the complexity and irregularity of modeled material distributions, [GT15] introduced material convolution surfaces (primitives) as an extension of the source-based model. A material convolution primitive is a scalar field function of a potential function for a surface primitive (such as point, straight line segment, spline, plane). It provides the means to evaluate the material composition at any given point within the area of primitive influence. This field function for the given primitive is defined by the convolution integral of a binary point membership function defining the material primitive geometry with the "material potential function", which is some typical convolution kernel. The control features of this model in terms of both geometry and materials are discussed in [GT17], which allow the designer to modify material distributions with the purpose of achieving necessary material compositions.

Material composition in [SG17] is also defined as a function of distance from some material reference entity. The geometric object is decomposed using the Medial Axis Transform (MAT). The user needs to specify material compositions at the boundary and at the MAT points. In the object interior, material compositions are interpolated using distance functions to these points. Note that these formulations are quite straightforward for 2D shapes and are much more complicated for the $3 \mathrm{D}$ case.

The work [LJJ*17] presents a set of primitive material attribute functions and constructive operations to create $3 \mathrm{D}$ solid textures. First, space partitions for attributes are created using geometric primitives such as spheres, cylinders and others. Then, various deformations are applied to create a complex texture. These deformations are controlled by points and straight lines with associated asymmetric and oscillating displacement functions. Genetic operations on created textures are also supported such as random alteration (mutation), functions swapping (cross-over) and others.

The work [AW17] describes a specific operation on creating a transition of a material property interpolated between given values within a prescribed transition region of arbitrary geometry and within the given tolerance. Examples of transition functions are given in the linear, trigonometric and polynomial forms in the Cartesian and cylindrical coordinates. The transfinite interpolation was mentioned by the authors, but not applied in the examples.

\subsubsection{Fully procedural models}

In some cases the above-mentioned models are not sufficient to describe material distribution due to some limitations such as difficulties to explicitly identify material features or primitives and operations for constructive modeling. In such a case, tools for fully procedural modeling have to be involved that allow the user to create a procedure taking point coordinates as the minimal input and producing geometric and material property information as output. In some cases a procedure for geometry and material distribution generation followed by direct $3 \mathrm{D}$ printer control has to written in a general-purpose programming language [DMO15, BKWO16]. This is very labor intensive and requires involvement of highly skilled developers. We are interested here in different approaches and supporting tools such as special-purpose scripting languages or APIs in general-purpose languages allowing for the evaluation of scalar fields for both geometry and material properties.

In the general area of computer graphics, the research field called solid texturing [P85a, P85b, PCOS10] yielded a number of analytical functions describing objects internal appearance, namely spatial color variations, thus imitating volumetric structures of wood, marble and other complex materials irrespective of objects geometry. Solid texturing functions are compositions of the given basic functions such as pseudo-random solid noise defined by a series of nested trigonometric functions. This approach is further developed in [LJJ17] by deriv- 
ing functions for solid textures as superpositions of basic analytical functions such as exponential and trigonometric ones. Solid texturing is supported in several computer graphics tools such as Renderman Shading Language [HL90], POV-Ray scripting language [PV17] and others.

In a volume modeling setting, extending solid texturing [PH89] introduced base level density modulation functions and their combinations to represent various volumetric natural materials and phenomena with the technique called hypertexturing. This approach was adopted in [PCB99] to modeling spatially varying material distributions with gradients of density modulation functions. The gradient information is controlled interactively using several basic functions, polynomial or spline interpolations of discrete data. These approaches can be considered early steps towards fully procedural generation of volumetric material distributions.

Procedural geometry generation with scalar fields was introduced in the HyperFun language fully supporting FRep [ACF*99]. Then, it was extended to heterogeneous volume modeling in [PASSO1], [CAP*05], where special arrays can be defined for volumetric material properties. While defining such arrays, full procedural definitions can be used thus introducing locally or globally varying FGM.

In $\left[\mathrm{CDM}^{*} \mathrm{O} 2\right]$ a special-purpose scripting language was also introduced for authoring complex volumetric models by specifying both geometric and material properties. The overall object geometry and boundaries between materials are defined with surface meshes converted to continuous distance fields. Each layer is assigned a material type and thickness, which can very procedurally. For example, thickness can be controlled by adding randomness or turbulence to the distance field. Material properties such as density or color can be modified procedurally within the script. Some constructive operations such as scale, union, intersection are also supported.

The work [VWRM13] presents an approach and programming tools for procedurally specifying geometric and material properties for objects to be $3 \mathrm{D}$ printed. A special-purpose $\mathrm{C}$-like programming language OpenFL is introduced and its compiler is implemented. The language allows for continuous volumetric material definition evaluated procedurally upon the request based on the distance field to the object surface represented by a mesh. An example of an OpenFL library for procedurally specifying complex materials can be found in [W13]. Such a framework is hard to operate for a non-programming user and an intuitive graphical user interface is needed for them. The authors addressed this issue in [VKWM16] and presented an interactive system called Foundry for designing spatially varying material properties. The interface of the system is built around the graph serving operator as a visual representation of the object generation procedure. Nodes of the graph represent operators and edges represent the dataflow. The operators allow for decomposing space into partitions on the basis of distances from surfaces and other sources; for applying some geometric transformations, and for assigning materials to spatial partitions. The range of available materials includes dual material composites and cellular microstructures, FGMs and biomimetic materials are based on sampled data. Each change made in the operator graph is followed by compilation of the currently designed procedure to its OpenFL-based description.

A hybrid procedural representation is proposed in [MUSA15] for parametric trivariate solids and attributes describing their material and other physical properties. Set operations can be applied to generated solids with attributes [MSA12]. The weights for attributes are computed using SDFs for primitives with maintaining the partition of unity requirement. To practically apply set operations, primitives are also converted to SDFs and R-functions are applied to construct SDF of a complex solid. A declarative programming language is introduced, which is parsed and complied to Java for the further evaluation.

In general, procedural models provide most powerful and flexible means for material definition embracing most of other methods. They are essentially hardware independent and resolution independent. However, the procedural models need special user training to be 
used efficiently and require in general additional GUI understanding for non-programming users.

\section{Challenges of multi-material design and fabrication}

In addition to the technical challenges of heterogeneous object multi-material (HOM) and heterogeneous object (HO) representation, a range of key challenges remain before HOM can be adopted as mainstream practice. Where a variety of solutions are emerging, many questions revolve around which are the most appropriate and useful methods to the scientific, engineering and art communities which will use them.

\subsection{Representation of materials}

The focus of this survey has been material distribution with continuous scalar fields. There are two general approaches for material representation: the classification of materials and properties as attributes; or the definition of material properties through microstructures. These correspond to compositional heterogeneity and structural heterogeneity, respectively at nano and micro / meso-scales [KTo7]. The key challenge to drive forward the adoption of HOM is the choice, compromise, or convergence of these approaches. Put another way, from an HO CAD system-builder's perspective, what is the best way to represent material properties? Should the end-user be working with and designing microstructure geometries or assigning material attributes?

While microstructures more closely resemble the atomic and molecular nature of materials $\left[\mathrm{SBA}^{*} 16\right]$, it could perhaps be argued that assigning material attributes is more intuitive from a user's perspective. As we shall consider later in this section, there are also issues regarding rendering, interaction paradigm, analysis, fabrication and system integration.

It may be natural to consider the application domain and to choose an appropriate representation scheme. However, convergences and interactions between disciplines, as well as emerging fabrication techniques, could quickly render a system obsolete, inhibit workflow or simply prevent the production of relevant designs. Rather than choosing one or another, we pose an open research question to design a material description which operates interchangeably and seamlessly at different physical scales.

\subsection{Rendering}

The ability to visualise a HOM is clearly a vital aspect to widespread adoption. Challenges arise here primarily around the speed of rendering, especially at interactive framerates and for dynamic data, but more fundamental questions arise surrounding the most appropriate visualisation strategies to convey interior and multi-material information in an intuitive way for the user.

\subsubsection{Overview of existing rendering approaches}

Broadly, we can consider two relevant modes of rendering which we will term photorealistic and functional [Fo3a]. The purpose of photo-realism is to represent an object in a way that it is indistinguishable from a photograph and is therefore useful for final model visualisation. Functional realism presents the same visual information but in a way which clarifies relevant properties in perhaps a more diagrammatical format, such as displaying separate components in different bright colours. The functional realism approach is therefore of great relevance during the content creation process and in applications where it is beneficial to enhance the visualisation of certain aspects of a complex dataset, such as in medical visualisation [PBC16].

Visualisation may then be broken into two further categories: opaque and semitransparent. Opaque rendering largely involves the reduction of the $\mathrm{HO}$ to a BRep, for which rendering is traditionally well defined. Such conversion processes clearly depend on the na- 
ture of the HO representation method, but include the set of isosurface extraction methods such as marching cubes and marching tetrahedra [NYo6]. The greatest advantage of this approach is that BRep rendering, especially polygonal meshes, is accelerated by graphics hardware and is therefore possible at interactive framerates. Issues include conversion speed, loss of accuracy and potentially an increase in data size.

The primary challenge for rendering and analysis of HOMs, however, is to visualise not just the exterior boundary but also the interior material distribution. One method is to use clipping geometry to cut into the object and thus reveal interior properties on the intersecting surface. Planes or other non-planar surfaces may be used [EHK*06]. Such techniques allow for the analysis and accurate interpretation of material distributions, which is especially useful in medical and scientific applications [PB13]. However, they only allow for the visualisation of a small portion of the volume at any particular instance, which may inhibit understanding of the object as a whole.

Semi-transparent HOM rendering may be present as a mixture of transparent and opaque regions. It allows to visualised and analysed internal features and structures. A viewing ray through the volume must be integrated to find the overall light intensity. Common discretizations are based around ray marching, where the volume densities are taken at specific sample points and the illumination and optical density levels are accumulated [PH89].

For realistic rendering it is necessary to consider light that is absorbed, emitted and scattered along the viewing path. At each sample point a phase function is also evaluated to determine the local illumination based on the material properties. Shadows may be added with relative technical ease by marching toward light sources from each sample point. More physically accurate approaches must take into account the single and multiple scattering paths that light takes through an HO, for which a wide range of approaches exist [EHK ${ }^{*}$ 6] [JSYR14].

For a functional rendering approach, physical accuracy is not necessarily a priority, although many aspects are often incorporated. Arbitrary transfer functions are frequently used to determine the mapping between material properties and their optical characteristics, which may be adjusted to emphasise certain aspects [EHK $\left.{ }^{*} \mathrm{O} 6\right]\left[\mathrm{LKG}^{*} 16\right]\left[\mathrm{PBC}^{*} 16\right]$.

Advantages of semi-transparent rendering are that it potentially displays more information about the model and may be more realistic for some materials which allow to provide more precise analysis of the model features. Primary disadvantages include rendering speed and perceptual issues where views of the object are unclear or specific internal features are difficult to identify. This latter issue is dependent on use-case and is one of the primary motivators for a functional rendering approach.

\subsubsection{Key remaining challenges in rendering}

One of the primary disadvantages of semi-transparent HOM rendering is that it can be difficult to distinguish between regions in the volume, especially in depth. While this may not present an issue for relatively simple objects, in some contexts such as medical imaging, it becomes vital. An artist or engineer producing a HOM would need an accurate and effective visualization method that does not create excessive workload through mental demand and minimal effort.

A number of investigations have been conducted to examine user perceptions of volume rendering [JSYR14], especially within medical visualisation [PBC*16]. However, these studies mostly consider an observer's perspective rather than that of a creator so it is unclear how the requirements and results would map to a different use-case. The challenge therefore remains to identify the 'best' way of presenting a HOM to a content creator. We speculate that this will depend on the context and the discipline and that a variety of techniques may be beneficial.

While general ray marching based techniques may be used in real-time, the quality can be variable. HOMs are typically computationally expensive to sample, when compared to BRep, so relatively few samples may be necessary to maintain interactive framerates. This leads to a poor approximation of the volume rendering integral, potentially resulting in visual artefacts. 
Methods to accelerate this are usually limited to handling static geometry, which may be suitable for data obtained by sampling (e.g. medical MRI, CT) but not for fully dynamic data [GKT10]. Further, realistic rendering requires the handling of multiple scattering lighting interactions, again for which there are currently relatively few methods that work with dynamic data [SKPo9] [WWH*10] [CPZ12] [KMM*17]. More work is therefore needed to support quality rendering of dynamic HOMs.

HOM rendering typically involves significant processing power and memory requirements. While GPU speed and memory specifications continue to improve, data capture and user expectations also progress and there will likely always be datasets that exceed the capacity of commodity hardware. Furthermore, in recent years the speed at which data can be transferred has not kept up with the pace of memory capacity, so the increases cannot be fully utilised for HOM rendering [JSYR14]. The usual approach to rendering large datasets is to divide the data into working sets that fit onto the GPU and then combine the results in some way [EHK ${ }^{*}$ 06] [JSYR14] [BHP15]. The main issues are of scalability, but there is some indication that modern GPU ray-guided approaches offer better performance in this respect [BHP15].

Once it becomes clear which material representation method to pursue, the rendering approach naturally must follow. Materials may be reproduced in realistic or functional manners, usually through the application of measured real-world samples [HS17]. However, a HOM system may be used to define unusual composites which could be difficult to reproduce in the real world, or for which there is limited material information. It is currently unclear how this could be handled in a general sense.

The inclusion of microstructures may present speed costs based on sampling resolution, which would depend on the underlying geometry representation method. However, more fundamental issues will become apparent as the physical scale reduces to the point where features are of comparable size to the wavelength of visible light. At such a point, realistic rendering must take into account the effects of diffraction and interference, which can be especially difficult in real-time [DTS*14] [TG17].

\subsection{Creative modeling: content creation}

Content creation is the process of interactively constructing a $3 \mathrm{D}$ model by an artist, designer or engineer and is currently poorly defined for HOM [GZR*15]. In addition to rendering at interactive framerates, this process requires an intuitive interface and an appropriate paradigm for modeling. For boundary definition a number of modeling paradigms exist within the current realm of digital $3 \mathrm{D}$ content creation: sculptural (e.g. ZBrush), constructive modeling (e.g., CSG), surface forming, and deformations. Each paradigm is relatively well established and their suitability for particular content types is generally accepted among a broad range of communities. For HOM, the communities are still young, relatively few tools exist and common modeling paradigms are not yet established.

Within HOM the creation problem is not limited to the object's boundary but also the interior material distribution and potentially microstructure formation. As we have seen in this review, a wide variety of approaches have been developed for specifying interior properties. The primary open question regarding content creation is the identification and categorisation of the 'best' way for a designer to create a heterogeneous volume model. While boundary formation is well established there are currently no commonly accepted paradigms for interior material modeling. An increase in tool availability and familiarity by content creators will naturally result in the emergence and domination of appropriate paradigms. To provide some insights, future studies could undertake an in-depth examination of the classes of design problem that HOMs can solve, consider how designers are currently attempting to solve these problems and conduct a detailed examination of their requirements. Following the trend of boundary modeling, the results would likely follow the application domain. An engineer 
would be expected to have very different requirements to an artist, though some overlap may exist.

\subsection{Fabrication (CAM)}

The potential real-world applications of heterogeneous volume modeling are vast, from aerospace to biology to decorative glass making. In a mathematical or virtual model it is relatively easy to specify multiple materials for a given volume in space, but the physical fabrication of models with such properties is not as straightforward. For the model to be brought into the physical world it must be fabricated, but traditional means are based on homogeneous materials or assemblies of multiple homogeneous parts. The development of FGMs in engineering sectors has expanded traditional techniques to include some heterogeneous fabrication capabilities.

Broadly, FGM fabrication processes have been categorised by [BSM14] into: constitutive, where layers of different material properties are built up; homogenizing, where a sharp interface is converted into a gradient; and segregation, which starts with a homogeneous material that is converted into a gradient. The most flexible of these is considered to be constitutive.

Constitutive fabrication methods can offer flexible control over the gradient and object geometries. Vapour deposition can be used for excellent microstructure control, but is limited to thin surface coatings [BSM14]. A range of casting approaches have been developed [SMD15], which allow smooth gradients but are limited to simple geometries. In powder metallurgy, powder mixtures are packed into layers and fused [EE15] and while good control of the gradient may be achieved in a step-wise fashion, there are again limitations to the geometries that can be handled. Freeform fabrication, also known in its general terms of additive manufacturing (AM) or $3 \mathrm{D}$ printing, is broadly able to fabricate both arbitrary gradients and geometries, but resolution remains an issue.

All such fabrication methods are still young and experimental when compared to the manufacturing powerhouses that drive our homogeneous-object-based industries. Active developments seek to reduce and mitigate their issues and to broaden their applicability. Here we shall outline a small subset of generally relevant fabrication challenges.

Material mixing as a process is relatively well defined within techniques such as powder metallurgy. However, while the advantage of AM is its potential for arbitrary gradients and geometries, one key challenge is to ensure adequate mixture of materials.

Discrete approaches of manufacturing generally involve specifying regions of different materials in close spatial proximity [CY08]. Dithering patterns can result in seemingly smoother gradients at a macro-scale [CSPTo3, LMP*04, ZXYo4, OKT11, VWRM13, BATU18], but these are naturally resolution dependent and still do not necessarily result in smooth transitions. Multiple plastic filaments may be fused together before deposition [SL17] though initial results are more akin to a discrete approach with distinct sublayers. In general, the material mixing problem remains an open challenge for AM processes.

As described in Introduction and section 3.1, there is an intimate connection between material and microstructure. The manufacture of microstructures with varying properties allows for the variation of mechanical properties [WZL*18]. It is therefore important that multimaterial fabrication is able to fully support microstructures.

One subset of techniques involves the direct control of the AM hardware in some way, to produce a procedural pattern at the native resolution [DMO15]. Techniques such as viscous thread instability [LL16] have been used to fabricate higher resolution structures than a printer's native resolution. Microstructure variation control has been investigated for metal AM [NCBR18] however, understanding of these processes is still at an early stage [GLD*16]. Techniques and applications of materials that change properties after their manufacture have also been reviewed as a new direction, with multi-material fabrication as a prerequisite [T14] [XSW17]. 
One of the major drawbacks of many of these techniques is that they are highly dependent on specific hardware or manufacturing technology, so the results may be difficult to transfer and model in the general sense. The broader adoption of HOs will require that any fabrication methods are reproducible with well-defined material properties [GZR ${ }^{*} 15$ ] and as such that their properties will match those predicted through analysis techniques.

It is of vital importance to engineering applications that manufactured materials can be relied upon for their physical properties. This means that the computer model must be fabricated into a real-world object in a consistent manner, such that its physical properties closely match with its predicted (modeled) properties. Critical components must undergo a battery of tests and inconsistencies can result in costly delays. Such requirements present key challenges for multi-material heterogeneous objects, especially considering that conventional measurement techniques are not well suited to test graded materials [BKT $\left.{ }^{*} 17\right]$. Dependencies on individual hardware, manufacturers, proprietary and closed technologies, or any other factor that implies no guarantee of at least medium-term continuation, are also unlikely to be conducive to widespread engineering adoption. The primary challenge then, is for the maturation of the experimental fabrication techniques into a reliable manufacturing industry, with an established workflow and appropriate set of standards.

\subsection{Challenges in system integration and architecture}

The primary open challenge is the development of a complete HO solution, which allows heterogeneous multi-material objects to be modeled, analysed and fabricated.

As identified in [WLWo8, GT15], substantial work has been undertaken in developing representation schemes for HOs but there is comparably little treatment of their integration into a full CAD environment. The majority of current commercial CAD systems are based on BRep or homogeneous volumes, but given the efforts required to build a CAD system most attempts at heterogeneous multi-material modeling are usually proposed as extensions to existing systems [GT15] or use existing systems for geometry definition [ZCFO5]. The requirements of $\mathrm{HO}$ modeling are not necessarily compatible with the system features provided by BRep CAD systems. HO modeling typically requires processing steps to evaluate geometry or material distribution which can lead to fundamentally different requirements for each subsystem that needs to sample or handle the model [QD03]. A number of researchers therefore do not advocate extending existing systems, but rather develop their own bespoke approaches [QDo3 WLWo8].

Analysis is an important stage in the modern modeling process and may generally be considered a prerequisite for adoption in engineering applications [STG11]. Existing techniques for forward integration (analysis) and inverse integration (shape and / or material optimisation) were reviewed in section 2.4.2 and the problem is generally considered an open one for HOs. Once solutions are found, they must be integrated into a system with appropriate workflow. Further, additional considerations include: how to handle microstructures and multiscale geometry [LS18]; the integration of $\mathrm{HO}$ analysis into a CAD/CAE system; the choice of appropriate interaction paradigm; and appropriate dataflow and file exchange formats that can handle complex engineering-scale multi-material HOs with microstructures.

Some attempts have been made at defining a feature set for HO systems, which we can expand upon as a result of our survey:

- Heterogeneous Multi-Material Object representation scheme must:

○ Be compact and exact

- Include material definition

- Operate interchangeably and seamlessly at different physical scales

- Support complex solids, both in geometry and material distribution

- Rendering support must:

○ Run at interactive speeds 
O Offer an intuitive and simultaneous visualisation of geometry, topology and material information

- Modeling approach must:

○ Be intuitive

○ Support definition and modification of geometry, topology and material distribution at multiple physical scales

- Dynamic models:

○ Must allow for spatial and time dependent material distributions

○ (This has implications across multiple sub-systems)

- CAX integration:

○ Analysis: forward integration as a minimum, preferably inverse integration to allow optimisation of shape and materials as part of workflow

- Shape and material properties must be fully available to CAE subsystem

- Compatible with industrial standards for data exchange.

\section{Conclusion}

This survey covers rapidly growing important area of multi-material modeling, rendering, visual analysis and fabrication. Techniques of software rendering, interactive design, and different types of fabrication are outlined. The main difficulty is outlined, namely lack of standards and supporting software tools, especially for AM. Requirements to new CAD software system are formulated.

\section{References}

[A12] Allaire, G. (2012). Shape optimization by the homogenization method (Vol. 146). Springer Science \& Business Media.

[ABC*0o] Armstrong, C., Bowyer, A., Cameron, S. et al., Djinn. A Geometric Interface for Solid Modeling, Information Geometers, Winchester, UK, 2000.

[ACF* 99] V. Adzhiev, R. Cartwright, E. Fausett, A. Ossipov, A. Pasko, V. Savchenko, HyperFun project: a framework for collaborative multidimensional F-rep modeling, Implicit Surfaces '99, Eurographics/ACM SIGGRAPH Workshop (Bordeaux, France, September 13-15 1999), J. Hughes and C. Schlick (Eds.), 1999, pp. 59-69.

[AKK*02] Adzhiev, V., Kartasheva, E., Kunii, T., Pasko, A., Schmitt, B.: Hybrid cellularfunctional modeling of heterogeneous objects, Journal of Computing and Information Science in Engineering, vol. 2, 2002, pp. 312-322.

[ASTM11] ASTM Standard. Standard Specification for Additive Manufacturing File Format (AMF), version 1.1, July 2011.

[AW17] G. Ameta, P. Witherell, A novel transition region representation for additive manufacturing for graded materials, structures and tolerances, Proceedings of the ASME 2017 International Design Engineering Technical Conferences and Computers and Information in Engineering Conference IDETC/CIE 2017, Volume 1, paper No. DETC2017-68390, 2017, Voo1To2A013, 10 pages.

[Boo] Buhmann, M. D. (2000). Radial basis functions. Acta Numerica 2000, 9, 1-38

[Bo6] Belyaev, A. On transfinite barycentric coordinates. In Proceedings of the fourth Eurographics Symposium on Geometry Processing (pp. 89-99). Eurographics Association.

[B95] A. Bowyer, Svlis: Introduction and User Manual, Information Geometers, UK, $1995,128 \mathrm{p}$.

[BATU18] A. Brunton, C. Arikan, T. Tanksale, P. Urban, 3D Printing Spatially Varying Color and Translucency, ACM Transactions on Graphics, Volume 37 Issue 4, August 2018, Article No.157

[BFP13] A. Belyaev, P.-A. Fayolle, A. Pasko, Signed Lp-distance fields, Computer-Aided Design, Volume 45, Issue 2, February 2013, pp. 523-528. 
[BFSSo8] Biswas, A., Fenves, S. J., Shapiro, V., and Sriram, R., 2008, "Representation of Heterogeneous Material Properties in the Core Product Model,” Eng. Comput.,24, pp. 43-58.

[BHP15] J. Beyer, M. Hadwiger, H. Pfister. 2015. State-of-the-Art in GPU-Based LargeScale Volume Visualization. Comput. Graph. Forum 34, 8 (December 2015), 13-37. DOI: https://doi.org/10.1111/cgf.12605

[BKK*15] L. Branets, V. Kubyak, E. Kartasheva, V. Shmyrov, D. Kandybor, Capturing geologic complexity in a simulation grid, SPE Reservoir Simulation Symposium (23-25 February, Houston, Texas, USA), SPE-173270-MS, Society of Petroleum Engineers, 2015.

[BKT $\left.{ }^{*} 17\right]$ Valmik Bhavar, Prakash Kattire, Sandeep Thakare, Sachin patil and Dr. RKP Singh, 2017. A Review on Functionally Gradient Materials (FGMs) and Their Applications. IOP Conf. Ser.: Mater. Sci. Eng. 229012021

[BKWO16] Christoph Bader, Dominik Kolb, James C. Weaver, Neri Oxman, Data-driven material modeling with functional advection for $3 \mathrm{D}$ printing of materially heterogeneous objects, 3D Printing and Additive Manufacturing. Jun 2016, 3(2): 71-79.

[BSDoo] Bhashyam S, Shin KH, Dutta D. An integrated CAD system for design of heterogeneous objects. Rapid Prototyping J 2000;6(2):119-35.

[BSM14] Bohidar, S.K., Sharma, R. and Mishra, P.R., 2014. Functionally graded materials: A critical review. International Journal of Research, 1(4), pp.289-301.

[BSTo4] A. Biswas, V. Shapiro, and I. Tsukanov, Heterogeneous material modeling with distance fields, Computer Aided Geometric Design, vol. 21, no. 3, 2004, pp. 215-242.

[BTG15] E. Bohl, O. Terraz, D. Ghazanfarpour, Modeling fruits and their internal structure using parametric 3Gmap L-systems, The Visual Computer, Volume 31, Issue 6-8, 2015, pp. 819-829.

[BS17] T. Brochu, R. Schmidt, Geometric Modeling of Multi-Material Printed Objects, Eurographics 2017, Short Papers, A. Peytavie and C. Bosch (Eds.).

[CAP*05] R. Cartwright, V. Adzhiev, A. Pasko, Y. Goto, T. Kunii, Web-based shape modeling with HyperFun, IEEE Computer Graphics and Applications, vol. 25, No. 2, 2005, pp. 60-69.

[CDM*02] Cutler, B., Dorsey, J., McMillan, L., Muller, M., Jagnow, R.: A procedural approach to authoring solid models. In: ACM SIGGRAPH, pp. $302\{311$ (2002)

[CCZ16] Mingtao Cui, Hongfang Chen, Jingling Zhou, A level-set based multi-material topology optimization method using a reaction diffusion equation, Computer-Aided Design 73 (2016) 41-52.

[CLD*13] D. Chen, D. I. W. Levin, P. Didyk, P. Sitthi-amorn, W. Matusik, Spec2Fab: A Reducer-Tuner Model for Translating Specifications to 3D Prints, ACM Transactions on Graphics (SIGGRAPH 2013), Volume 32 Issue 4, July 2013, Article No. 135

[CPZ12] Chen, G., Peers, P., Zhang, J. et al. 2012. Real-time rendering of deformable heterogeneous translucent objects using multiresolution splatting. Visual Computer (2012) 28: 701. https://doi.org/10.1007/s00371-012-0704-1

[CSo8] Jiaqin Chen, V. Shapiro, Optimization of continuous heterogeneous models, Heterogeneous Objects Modeling and Applications, Lecture Notes in Computer Science, Volume 4889, 2008, pp. 193-213.

[CSPTo3] Cho W, Sachs EM, Patrikalakis NM, Troxel DE. A dithering algorithm for local composition control with three-dimensional printing. Comput Aided Des 2003;35: 851-867.

[CToo] Chiu WK, Tan ST. Multiple material objects; from CAD representation to data format for rapid prototyping. Comput Aided Des 2000;32:707-17.

[CTGF15] F. Conde-Rodríguez, J.-C. Torres-Cantero, Á.-L. García-Fernández, F.-R. FeitoHigueruela, A Comprehensive framework for modeling heterogeneous objects, The Visual Computer, 08/2015

[CWW13] K. Crane, C. Weischedel, M. Wardetzky, Geodesics in Heat: A New Approach to Computing Distance Based on Heat Flow, ACM Transactions on Graphics 32(5), 2013. 
[CYo8] Chiu WK, Yu KM. Direct digital manufacturing of three-dimensional functionally graded material objects, Comput Aided Des 2008;40:1080-1093.

[DFo9] Dyken, C., \& Floater, M. S. (2009). Transfinite mean value interpolation. Computer Aided Geometric Design, 26(1), 117-134.

[DMO15] J. Duro-Royo, L. Mogas-Soldevila, N. Oxman, Flow-based fabrication: An integrated computational workflow for design and digital additive manufacturing of multifunctional heterogeneously structured objects, CAD, 2015

[DTD*15] E.L. Doubrovski, E.Y. Tsai, D. Dikovsky, J.M.P. Geraedts, H. Herr, N. Oxman, Voxel-based fabrication through material property mapping: A design method for bitmap printing, Computer-Aided Design, Volume 60, Pages 3-13 (March 2015)

[DTS*14] Dhillon, D., Teyssier, J., Single, M., Gaponenko, I., Milinkovitch, M., Zwicker, M. (2014), Interactive Diffraction from Biological Nanostructures. Computer Graphics Forum, 33: 177-188. doi:10.1111/cgf.12425

[DZKo6] R. Dwivedi, S Zekovic, R. Kovacevic, Field feature detection and morphingbased process planning for fabrication of geometries and composition control for functionally graded materials, Journal of Engineering Manufacture 220(10):1647-1661, 2006.

[EE15] El-Wazery, M.S. and El-Desouky, A.R., 2015. A review on functionally graded ceramic-metal materials. Journal of Materials and Environmental Science, 6(5), pp.1369-1376.

[EHK*06] K. Engel, M. Hadwiger, J. Kniss, C. Rezk-salama, D. Weiskopf. 2006. RealTime Volume Graphics. A. K. Peters, Ltd., Natick, MA, USA.

[Fo3a] Ferwerda, James. (2003). Three Varieties of Realism in Computer Graphics. Proceedings of SPIE - The International Society for Optical Engineering. 5007. 10.1117/12.473899.

[Fo3b] Floater, M. S. (2003). Mean value coordinates. Computer aided geometric design, 20(1), 19-27.

[FKRo5] Floater, M. S., Kós, G., \& Reimers, M. (2005). Mean value coordinates in 3D. Computer Aided Geometric Design, 22(7), 623-631.

[FPA11] O. Fryazinov, A. Pasko, V. Adzhiev, BSP-fields: An exact representation of polygonal objects by differentiable scalar fields based on binary space partitioning, ComputerAided Design, vol. 43, no. 3, March 2011, pp. 265-277.

[FPSMo6] P.-A. Fayolle, A. Pasko, B. Schmitt, N. Mirenkov, Constructive heterogeneous object modeling using signed approximate real distance functions, Journal of Computing and Information Science in Engineering, ASME Transactions, Vol. 6, No. 3, September 2006, pp. 221-229.

[FSP15] O. Fryazinov, M. Sanchez, A. Pasko, Shape conforming volumetric interpolation with interior distances, Computers \& Graphics, vol. 46, 2015, pp. 149-155.

[FSTo6] M. Freytag, V. Shapiro, I. Tsukanov, Field modeling with sampled distances, Computer-Aided Design 38 (2006) 87-100.

[FVP13] O. Fryazinov, T. Vilbrandt, A. Pasko, Multi-scale space-variant FRep cellular structures, Computer-Aided Design, Volume 45, Issue 1, 2013, pp. 26-34.

[GLD*16] Galarraga, H., Lados, D.A., Dehoff, R.R., Kirka, M.M., Nandwana, P. Effects of the microstructure and porosity on properties of Ti-6Al-4V ELI alloy fabricated by electron beam melting (EBM) (2016) Additive Manufacturing, 10, pp. 47-57.

[GKT10] V. Gupta, K. Kasana, P. Tandon, Computer Aided Design Modeling for Heterogeneous Objects, International Journal of Computer Science Issues, Vol. 7, Issue 2, No 5, March 2010, pp. 31-38.

[GKT12] Gupta V, Kasana KS, Tandon P. Reference based geometric modeling for heterogeneous objects. Comput Aided Des Appl 2012;9(2):155-65.

[GRP12] Olya Grove, Khairan Rajab and Les A Piegl, Heterogeneous modeling of medical image data using B-spline functions, Proc IMechE, Part H: J Engineering in Medicine 226(10), 2012, 737-751 
[GT15] V. Gupta, P. Tandon, Heterogeneous object modeling with material convolution surfaces, Computer-Aided Design, Volume 62, May 2015, Pages 236-247

[GT17] V. Gupta, P. Tandon, Heterogeneous Composition Adaptation With Material Convolution Control Features, J. Comput. Inf. Sci. Eng 17(2), 021008 (Feb 16, 2017)

[GVo7] Goupee, A. J., \& Vel, S. S. (2007). Multi-objective optimization of functionally graded materials with temperature-dependent material properties. Materials \& design, 28(6), 1861-1879.

[GW74] Gordon, W. J., \& Wixom, J. A. (1974). Pseudo-harmonic interpolation on convex domains. SIAM Journal on Numerical Analysis, 11(5), 909-933.

[GWB*12] Xiuli Gai, Xiao-hui Wu, L. Branets, K. Sementell, G. Robertson, ConceptBased Geologic Modeling Using Function Form Representation, SPE-161795-MS, Abu Dhabi International Petroleum Conference and Exhibition, Society of Petroleum Engineers, Abu Dhabi, UAE, Abu Dhabi International Petroleum Conference and Exhibition, Abu Dhabi, UAE, 2012.

[GZR*15] Wei Gao, Yunbo Zhang, D. Ramanujan, K. Ramani, Yong Chen, C. Williams, C. C.L. Wang, Yung C. Shin, Song Zhang, P. Zavattieri, The status, challenges, and future of additive manufacturing in engineering, Computer-Aided Design 69, December 2015, pp. 65-89

[H98] C. M. Hoffmann, Geometric and Solid Modeling. Morgan Kaufmann Pub, 1998, $337 \mathrm{pp}$.

[HFBGo2] Huang J, Fadel GM, Blouin VY, Grujicic M. Bi-objective optimization design of functionally gradient materials. Mater Des 2002;23:657.

[HLo9a] Hiller, J., Lipson, H., STL 2.0: a Proposal for a Universal Multi-Material Additive Manufacturing File Format, Solid Freeform Fabrication Symposium (SFF'o9), Austin, TX, USA, 2009, pp. 266-278

[HLogb] Hiller, J., Lipson, H., Design automation for multi-material printing

[HLo9c] Hiller J, Lipson H. Design and analysis of digital materials for physical $3 \mathrm{D}$ voxel printing. Rapid Prototyp J 2009;15:137-49.

[HMM11] J.Y. Hascoet, P. Muller, P. Mognol, Manufacturing of complex parts with continuous functionally graded materials (FGM), International Solid Freeform Fabrication Symposium, University of Texas at Austin, USA, 2011, pp. 557-569.

[HP14] HP Multi Jet Fusion technology, Technical White Paper, 4AA5-5472ENW, Hewlett-Packard, October 2014.

[HS99] Hiyoshi, H., \& Sugihara, K. (1999). Two generalizations of an interpolant based on Voronoi diagrams. International Journal of Shape Modeling, 5(02), 219-231.

[HSoo] Hiyoshi, H., \& Sugihara, K. (2000, May). Voronoi-based interpolation with higher continuity. In Proceedings of the sixteenth annual symposium on Computational geometry (pp. 242-250). ACM.

[HS17] Heinzl, C. and Stappen, S. (2017), STAR: Visual Computing in Materials Science. Computer Graphics Forum, 36: 647-666. doi:10.1111/cgf.13214

[HSS14] Hoffmann C., Shapiro V., Srinivasan V., Geometric interoperability via queries, Comput Aided Des 2014;46:148-59.

[HW13] I. Horváth and Y. Wang (Eds.), Computer-aided multi-scale materials and product design, Special issue, Computer-Aided Design, Volume 45, Issue 1, 2013.

[HQ05] Hua J., He Y., Qin H., Trivariate simplex splines for inhomogeneous solid modeling in engineering design. Journal of Computing and Information Science in Engineering 5, 2 (2005), 149-157.

[Joo] T. Jackson, Analysis of Functionally Graded Material Object Representation Methods, PhD Thesis, MIT, 2000.

[JLP*99] Jackson T., Liu H., Patrikalakis N., Sachs E., Cima M., Modeling and designing functionally graded material components for fabrication with local composition control. Materials and Design 20, 2-3, (1999), pp. 63-75 
[JLWo7] Ju, T., Liepa, P., \& Warren, J. (2007). A general geometric construction of coordinates in a convex simplicial polytope. Computer Aided Geometric Design, 24(3), 161-178.

[JPSC98] Jackson, T. R., Patrikalakis, N. M., Sachs, E. M., and Cima, M. J., 1998, Modeling and Designing Components With Locally Controlled Composition, Proceedings of Solid Freeform Fabrication Symposium, Austin, TX.

[JSYR14] Jönsson, D. , Sundén, E. , Ynnerman, A. and Ropinski, T. (2014), A Survey of Volumetric Illumination Techniques for Interactive Volume Rendering. Computer Graphics Forum, 33: 27-51. doi:10.1111/cgf.12252

[JSW05] Ju T., Schaefer S. and Warren J., Mean value coordinates for closed triangular meshes. ACM Trans. Graph., 24, 2005, 561-566.

[KBDH99] V. Kumar, D. Burns, D. Dutta, C. Hoffmann, A framework for object modeling, Computer-Aided Design, vol. 31, No. 9, 1999, pp. 541-556.

[KD97] V. Kumar, D. Dutta, An approach to modeling multi-material objects, Fourth Symposium on Solid Modeling and Applications, ACM SIGGRAPH, 1997, pp. 336-345.

[KD98] Kumar, V. and D. Dutta, "An Approach to Modeling and Representation of Heterogeneous Objects," ASME J of Mechanical Design, 120:4, 659-667, Dec 1998.

[KFC*07] Kopf, J., Fu, C. W., Cohen-Or, D., Deussen, O., Lischinski, D., \& Wong, T. T. (2007). Solid texture synthesis from 2d exemplars. ACM Transactions on Graphics (TOG), 26(3), 2.

[KMM*17] S. Kallweit, T. Müller, B. Mcwilliams, M. Gross, J. Novák. 2017. Deep scattering: rendering atmospheric clouds with radiance-predicting neural networks. ACM Trans. Graph. 36, 6, Article 231 (November 2017), 11 pages. DOI: https://doi.org/10.1145/3130800.3130880

[KPT12] X.Y. Kou, G.T. Parks, S.T. Tan, Optimal design of functionally graded materials using a procedural model and particle swarm optimization, Computer-Aided Design, Volume 44, Issue 4, April 2012, Pages 300-310.

[KTo5] Kou, X.Y. and S.T. Tan, A hierarchical representation for heterogeneous object modeling. Computer-Aided Design, 2005 - 37(3): p. 307-319.

[KTo7] Kou XY, Tan ST. Heterogeneous object modeling: A review, Comput Aided Design 2007;39:284-301.

[KTo8] Kou, X.,Tan, S., Heterogeneous object design: an integrated CAX perspective, in Heterogeneous Objects Modelling and Applications, Lecture Notes in Computer Science, vol 4889, Springer, 2008, pp. 42-59.

[LFAB14] Shuangyan Lei, M. Frank, D. Anderson, T. Brown, A method to represent heterogeneous materials for rapid prototyping: the Matryoshka approach, Rapid Prototyping Journal, Vol. 20, Iss 5, 2014, pp. $390-402$

[LJJ*17] Shufang Lu, Xiaogang Jin, Aubrey Jaffer, Fei Gao, and Xiaoyang Mao, Solid Mathematical Marbling, IEEE Computer Graphics and Applications, 2017, 37(2): 90-98.

[LKG*16] Ljung, P. , Krüger, J. , Groller, E. , Hadwiger, M. , Hansen, C., Ynnerman, A. (2016), State of the Art in Transfer Functions for Direct Volume Rendering. Computer Graphics Forum, 35: 669-691. doi:10.1111/cgf.12934

[LL16] Lipton, Jeffrey I., Lipson, Hod, 2016, 3D Printing Variable Stiffness Foams Using Viscous Thread Instability, Nature Scientific Reports, 6, 29996. https://doi.org/10.1038/srep29996

[LMP*04] Liu, H., Maekawa, T., Patrikalakis, N. M., Sachs, E. M., \& Cho, W. (2004). Methods for feature-based design of heterogeneous solids. Computer-Aided Design, 36(12), 1141-1159.

[LS15] Xingchen Liu, V. Shapiro, Random heterogeneous materials via texture synthesis, Computational Materials Science, Volume 99, March 2015, pp. 177-189

[LS17] Xingchen Liu, V. Shapiro, Sample-Based Synthesis of Functionally Graded Material Structures, J. Comput. Inf. Sci. Eng 17(3), 031012, 2017, Paper No: JCISE-16-2054. 
[LS18] Xingchen Liu, V. Shapiro, Multiscale shape-material modeling by composition, Computer-Aided Design, Volume 102, September 2018, Pages 194-203.

[M15] Monolith User's Guide, Monolith, October 2015.

[ME16] F. Massarwi, G. Elber, A B-spline based Framework for Volumetric Object Modeling, Computer-Aided Design, 2016

[MKR ${ }^{*}$ 99] Functionally Graded Materials: Design, Processing and Applications, Y. Miyamoto, W. A. Kaysser, B. H. Rabin, A. Kawasaki, R. G. Ford (Eds.), Kluwer Academic Publishers, Boston, 1999.

[MRP95] Markworth AJ, Ramesh KS, Parks WP. Review - modeling studies applied to functionally graded materials. J Mater Sci, 1995;30:2183 -2193.

[MS95] Markworth, A. J., and Saunders, J. H., 1995, "A Model of Structure Optimization for a Functionally Graded Material,” Mat. Lett., 22, pp. 103-107.

[MSA12] Morgan O, Subbarayan G, Anderson D. A hybrid hierarchical procedure for composing trivariate NURBS solids. Comput-Aided Des Appl 2012;9(2): 215-26.

[MUSA15] O.T. Morgan, K. Upreti, G. Subbarayan, D.C. Anderson, HiGeoM: A symbolic framework for a unified function space representation of trivariate solids for isogeometric analysis, Computer-Aided Design, Volume 65, August 2015, Pages 34-50.

[NCBR18] S. Narra, R. Cunningham, J. Beuth, A. Rollett, Location specific solidification microstructure control in electron beam melting of Ti-6Al-4V, Additive Manufacturing, Volume 19, 2018, Pages 160-166, ISSN 2214-8604, https://doi.org/10.1016/j.addma.2017.10.003.

[NSN*87] Niino M., Suzuki A., Nirai T., Watanabe R., Hirano T., Kuroishi N., Method of producing a functionally gradient material. Auszüge aus den Europäischen Patentanmeldungen, Teil I, Thomson Reuters, München, 1987;3(30):1555.

[NYo6] T. Newman, Hong Yi, A survey of the marching cubes algorithm, Computers \& Graphics, Volume 30, Issue 5, 2006, Pages 854-879, https://doi.org/10.1016/j.cag.2006.07.021.

[OBATSo3] Ohtake, Y., Belyaev, A., Alexa, M., Turk, G., \& Seidel, H. P., Multi-level partition of unity implicits. ACM Transaction on Graphics, 2003:22(3): 463-470.

[OC14] Objet50o Connex3, White paper, Stratasys, 2014, 7 pp.

[OFo6] Osher, S., \& Fedkiw, R. (2006). Level set methods and dynamic implicit surfaces (Vol. 153). Springer Science \& Business Media.

[OK11] I.T. Ozbolat, B. Koc, Multi-directional blending for heterogeneous objects, Computer-Aided Design 43.8 (2011): 863-875.

[OKT11] Oxman, N., Keating, S., and Tsai, E. Functionally graded rapid prototyping. In Proceedings of VRAP: Advanced Research in Virtual and Rapid Prototyping, P. Bartolo, Ed. (2011).

[Poo] Pratt MJ., Modeling of material property variation for layered manufacturing. The Mathematics of Surfaces, vol. IX. Cipolla R, Martin R (Eds), Springer; 2000. p. 486-500.

[P85a] Peachey, D. Solid texturing of complex surfaces. Computer Graphics 19(3), (Proc. SIGGRAPH 85), July 1985, pp. 279-286.

[P85b] Perlin, K. An image synthesizer. Computer Graphics 19(3), (Proc. SIGGRAPH 85), July 1985, pp. 287-296.

[PACo8] Pasko A., Adzhiev V., Comninos P. (Eds.), Heterogeneous Objects Modeling and Applications, Collection of Papers on Foundations and Practice, Lecture Notes in Computer Science, vol. 4889, Springer Berlin / Heidelberg, 2008, 285 p.

[PASS95] Pasko A., Adzhiev V., Sourin A., Savchenko V., Function representation in geometric modeling: concepts, implementation and applications, The Visual Computer, vol.11, No.8, 1995, pp.429-446.

[PASSo1] Pasko A., Adzhiev V., Schmitt B., Schlick C., Constructive Hypervolume Modeling, Graphical Models, special issue on Volume Modeling, vol. 63, No. 6, November 2001, pp. 413-442. 
[PB13] Bernhard Preim and Charl P. Botha. 2013. Visual Computing for Medicine: Theory, Algorithms, and Applications (2 ed.). Morgan Kaufmann Publishers Inc., San Francisco, CA, USA.

[PBC*16] Preim, B. , Baer, A. , Cunningham, D. , Isenberg, T. and Ropinski, T. (2016), A Survey of Perceptually Motivated $3 \mathrm{D}$ Visualization of Medical Image Data. Computer Graphics Forum, 35: 501-525. doi:10.1111/cgf.12927

[PCo3] Pratap A., Crawford R., Implementation of a gradient material modeling and design system, Proceedings of the 14th Solid Freeform Fabrication Symposium, Austin, TX 2003, 2003, pp. 150-161.

[PCB99] Park SM, Crawford RH, Beaman JJ., Functionally Gradient Material Design and Modeling Using Hypertexture for Solid Freeform Fabrication, Solid Freeform Fabrication Symposium, Austin, USA, 1999, p. 199-207.

[PCBoo] Park SM, Crawford RH, Beaman JJ., Functionally gradient material representation by volumetric multi-texturing for solid freeform fabrication. Proceedings of the 11th Solid Freeform Fabrication Symposium, Austin, USA, 2000, p. 350-61.

[PCOS10] N. Pietroni, P. Cignoni, M. Otaduy, R. Scopigno, Solid-Texture Synthesis: A Survey, IEEE Computer Graphics and Applications, vol. 30, July-August 2010, pp. 74-89.

[PDB*0o] Patil, L., Dutta, D., Bhatt, A. D., Jurrens, K., Lyons, K., Pratt, M. J., Sriram, R. D., Representation of heterogeneous objects in ISO 10303 (STEP), ASME International Mechanical Engineering Congress and Exposition (Orlando, Florida), 2000.

[PFV*11] A. Pasko, O. Fryazinov, T. Vilbrandt, P.-A. Fayolle, and V. Adzhiev, Procedural function-based modeling of volumetric microstructures, Graphical Models, vol. 73, no. 5, pp. 165- 181, 2011.

[PH89] Perlin, K., Hoffert, E. M., Hypertexture. In Proceedings of the 16th Annual Conference on Computer Graphics and Interactive Techniques, ACM, New York, NY, USA, SIGGRAPH '89, 1989, p. 253-262.

[PKM13] Panchal, J. H., Kalidindi, S. R., McDowell, D. L, "Key computational modeling issues in Integrated Computational Materials Engineering", CAD, 45(1), 2013, 4-25.

[PSo5] Pasko A., Shapiro V. (Eds.), Heterogeneous object models and their applications, Special Issue, Computer-Aided Design, vol. 37, issue 3, 2005.

[PV17] The Persistence of Vision Raytracer Homepage, URL: http://www.povray.org, accessed in September 2017

[QDo3] Qian X., Dutta D., Physics-based modeling for heterogeneous objects, Journal of Mechanical Design, Transactions of the ASME 125, 3 (2003), pp. 416-427.

[QDo4] Qian, X., and Dutta, D., Feature-Based Design For Heterogeneous Objects, Computer-Aided Design, 36(12), 2004, 1263-78.

[QD98] Qian X., Dutta D., Features in Layered Manufacturing of Heterogeneous Objects, Solid Freeform Fabrication Symposium, Austin, USA, 1998, p. 689-696.

[RLFo9] R. Rustamov, Y. Lipman, T. Funkhouser, Interior distance using barycentric coordinates, Computer Graphics Forum (Symposium on Geometry Processing), July 2009

[RO90] Rossignac, J., O'Connor, M., SGC: A dimension independent model for pointsets with Internal structures and incomplete boundaries, Geometric Modeling for Product Engineering, Ed. by M. Wozny, J. Turner, K. Preiss, North-Holland, 1990, pp. 145-180.

[RSZNo4] Rossl, C., Seidel, H. P., Zeilfeider, F., \& Nurnberger, G. (2004). Reconstruction of volume data with quadratic super splines. IEEE Transactions on visualization and Computer Graphics, 10(4), 397-409.

[RRSS16] W. Regli, J. Rossignac, V. Shapiro, V. Srinivasan, The new frontiers in computational modeling of material structures, Computer-Aided Design 77 (2016) 73-85.

[RSSTo1] V. Rvachev, T. Sheiko, V. Shapiro, I. Tsukanov, Transfinite interpolation over implicitly defined sets, Computer Aided Geometric Design, vol. 18, no. 3, 2001, pp. 195 - 220.

[So7] V. Shapiro, Semi-analytic geometry with R-functions, Acta Numerica, vol. 16, pp. 239-303, 2007. 
[S16] G. Schmitz, Microstructure Modeling in Integrated Computational Materials Engineering (ICME) Settings: Can HDF5 Provide the Basis for an Emerging Standard for Describing Microstructures?, The Journal of The Minerals, Metals \& Materials Society, Volume 68, Number 1, 2016, pp. 77-83.

[S68] Shepard, D. (1968, January). A two-dimensional interpolation function for irregularly-spaced data. In Proceedings of the 1968 23rd ACM national conference (pp. 517-524). ACM.

[S81] Sibson, R. (1981). A brief description of natural neighbour interpolation. Interpreting multivariate data, 21, 21-36.

[S99] Sethian J. A. Level Set Methods and Fast Marching Methods, 2nd ed. Cambridge University Press, Cambridge, United Kingdom, 1999.

[SBA*16] G.J.Schmitz, B.Böttger, M.Apel, J.Eiken, G.Laschet, R.Altenfeld, R.Berger, G.Boussinot, A.Viardin, Towards a Metadata Keyword Scheme for the Description of Materials - Keywords for the Description of Microstructures, Science and Technology of Advanced Materials, Taylor \& Francis, 2016 (to appear)

[SDo1] Shin KH, Dutta D. Constructive representation of heterogeneous objects. Trans ASME, J Comput Inform Sci Eng 2001;1(3):205-17.

[SFA*15] M. Sanchez, O. Fryazinov, V Adzhiev, P. Comninos, A. Pasko, Space-time transfinite interpolation of volumetric material properties, IEEE Tranactions on Visualization and Computer Graphics, vol. 21, No. 2, February 2015, pp. 278-288.

[SFP12] M. Sanchez, O. Fryazinov, A. Pasko, Efficient evaluation of continuous signed distance to a polygonal mesh, SCCG '12, Proceedings of the 28th Spring Conference on Computer Graphics, ACM Press, New York, 2012, pp. 101-108.

[SFFP15] M. Sanchez, O. Fryazinov, P.-A. Fayolle, A. Pasko, Convolution filtering of continuous signed distance fields for polygonal meshes, Computer Graphics Forum, vol. 34, No. 6, September 2015, pp. 277-288.

[SG17] G.K. Sharma \& B. Gurumoorthy, A hybrid approach to define and represent material distribution in heterogeneous objects, Computer-Aided Design and Applications, vol. 14, No. 1, 2017, pp. 70-82.

[SKo4] Samanta, K., and Koc, B., 2004, "Heterogeneous Object Design With Material Feature Blending," Comput.-Aided Des. and Appl., 1(1-4), pp. 429-439.

[SKo5] Samanta K, Koc B. Feature-based design and material blending for free-form heterogeneous object modeling. Computer-Aided Design 2005;37:287-305.

[SKo8] K. Samanta, B. Koc, Feature-based material blending for heterogeneous object modeling, in Heterogeneous Objects Modeling and Applications, Lecture Notes in Computer Science, vol. 4889, Springer Berlin / Heidelberg, 2008, pp. 142-166.

[SK16] V. Skorkovska, I. Kolingerová, Complex multi-material approach for dynamic simulations, Computers \& Graphics, 56 (2016), 11-19.

[SKP09] Shah, M.A., Konttinen, J. and Pattanaik, S., 2009. Image-space subsurface scattering for interactive rendering of deformable translucent objects. IEEE computer graphics and applications, 29(1).

[SL17] Hai-Chuan Song, Sylvain Lefebvre, Colored fused filament fabrication. $\underline{\text { CoRR }}$ abs/1709.09689 (2017) https://arxiv.org/abs/1709.09689

[SMD15] Saiyathibrahim.A, Mohamed Nazirudeen.S.S, Dhanapal.P. "Processing Techniques of Functionally Graded Materials - A Review." International Conference on Systems, Science, Control, Communication, Engineering and Technology (2015): 98-105.

[SMSBo1] Sukumar, N., Moran, B., Yu Semenov, A., \& Belikov, V. V. (2001). Natural neighbour Galerkin methods. International journal for numerical methods in engineering, 5O(1), 1-27.

[SPSo4] Schmitt B, Pasko A, Schlick C. Constructive sculpting of heterogeneous volumetric objects using trivariate B-splines. The Visual Computer 2004; 20(2-3):130-48. 
[STo2a] Siu Y. and Tan S., Source-based heterogeneous solid modeling, Computer-Aided Design, 34(1), 2002, $41-55$.

[STo2b] Y. Siu and S. Tan, Modeling the material grading and structures of heterogeneous objects for layered manufacturing, Computer-Aided Design, vol. 34, no. 10, pp. 705 716, 2002.

[STG11] V. Shapiro, I. Tsukanov, A. Grishin, Geometric issues in computer aided design/ computer aided engineering integration. J Comput Inf Sci Eng 2011, vol. 11/ 021005.

[T14] Tibbits, S. (2014), 4D Printing: Multi-Material Shape Change. Archit Design, 84: 116-121. doi:10.1002/ad.1710

[TG17] A. Toisoul and A. Ghosh. 2017. Real time rendering of realistic surface diffraction with low rank factorisation. In ACM SIGGRAPH 2017 Posters (SIGGRAPH '17). ACM, New York, NY, USA, Article 45, 2 pages. DOI: https://doi.org/10.1145/3102163.3102188

[TOIIo8] K. Takayama, M. Okabe, T. Ijiri, T. Igarashi, Lapped solid textures: filling a model with anisotropic textures, SIGGRAPH'08, ACM Transactions on Graphics, Volume 27, Issue 3 (August 2008), Article No. 53.

[TRSo4] I. Tobor, P. Reuter, C. Schlick, Multi-scale reconstruction of implicit surfaces with attributes from large unorganized point sets, Proceedings of Shape Modeling International, IEEE Computer Society, 2004, pp. 19-30.

[TSo5] Tsukanov, I., and Shapiro, V., 2005, Meshfree Modeling and Analysis of Physical Fields in Heterogeneous Media, Adv. Comput. Math., 23(1-2), pp. 95-124.

[TSNI10] K. Takayama, O. Sorkine-Hornung, A. Nealen, T. Igarashi, Volumetric modeling with diffusion surfaces, SIGGRAPH Asia, ACM Transactions on Graphics, Volume 29, Issue 6 (December 2010) Article No.: 180

[VKWM16] K. Vidimče, A. Kaspar, Ye Wang, W. Matusik, Foundry: hierarchical material design for multi-material fabrication, ACM User Interface Software and Technology Symposium (UIST), 2016.

[VWRM13] Vidimče, K., Wang, S. P., Ragan-Kelley, J., \& Matusik, W. (2013). OpenFab: A programmable pipeline for multi-material fabrication. ACM Transactions on Graphics (TOG), 32(4), 136.

[W13] Ye Wang, Fab Trees for Designing Complex 3D Printable Materials, Master's thesis, MIT, 2013, 68 pp.

[W75] Wachspress, E. L. (1975). A rational finite element basis. Elsevier.

[WBo9] Warkhedkar R., Bhatt A., Material-solid modeling of human body: A heterogeneous b-spline based approach, CAD Computer Aided Design 41, 8 (2009), 586-597.

[WLKZ15] Wang, Y., Luo, Z., Kang, Z., \& Zhang, N. (2015). A multi-material level setbased topology and shape optimization method. Computer Methods in Applied Mechanics and Engineering, 283, 1570-1586.

[WLWo8] Wu, X., Liu,W., Wang, M. Y., 2008. "A CAD modeling system for heterogeneous object”. Advances in Engineering Software, 39(5), pp. 444-453.

[WWo5] Wang MY, Wang XM. A level-set based variational method for design and optimization of heterogeneous objects. Comput Aided Des 2005;37(3): 321-37.

[WWH*10] Yajun Wang, Jiaping Wang, N. Holzschuch, K. Subr, Jun-Hai Yong, Baining Guo. 2010. Real-time Rendering of Heterogeneous Translucent Objects with Arbitrary Shapes. Computer Graphics Forum, Wiley, 2010, 29 (2), pp. 497-506. <10.1111/j.14678659.2009.01619.x>. <inria-00443634>

[WYZG11] Wang, L., Yu, Y., Zhou, K., Guo, B.: Multiscale vector volumes. ACM Trans. Graph. 30, 167 (2011)

[WZL*18] Xue Wang, Pu Zhang, Stephen Ludwick, Eric Belski, Albert C. To, Natural frequency optimization of $3 \mathrm{D}$ printed variable-density honeycomb structure via a homogenization-based approach, Additive Manufacturing, Volume 20, 2018, Pages 189-198, ISSN 22148604, https://doi.org/10.1016/j.addma.2017.10.001. 
[XSo5] Anping Xu, Leon L. Shaw, Equal distance offset approach to representing and process planning for solid freeform fabrication of functionally graded materials, ComputerAided Design 37 (2005) 1308-1318

[XSW17] Xin Li, Jianzhong Shang, Zhuo Wang, (2017) Intelligent materials: a review of applications in 4D printing, Assembly Automation, Vol. 37 Issue: 2, pp.170-185, https://doi.org/10.1108/AA-11-2015-093

[Y13] Dong-Jin Yoo, Heterogeneous object modeling using the radial basis functions, International Journal of Precision Engineering and Manufacturing, Vol. 14, No. 7, 2013, pp. 1133-1140.

[YQ07] Yang P., Qian X., A B-spline-based approach to heterogeneous objects design and analysis. CAD Computer Aided Design 39, 2 (2007), 95-111.

[YQZT14] Nan Yang, Zhi Quan, Dawei Zhang, Yanling Tian, Multi-morphology transition hybridization $\mathrm{CAD}$ design of minimal surface porous structures for use in tissue engineering, Computer-Aided Design, Volume 56, 2014, Pages 11-21.

[YYW12] Zhan Yuan, Yizhou Yu, and Wenping Wang. Object-space multiphase implicit functions. ACM Transactions on Graphics (TOG), 31(4):114, 2012.

[SG17] G.K. Sharma \& B. Gurumoorthy, A hybrid approach to define and represent material distribution in heterogeneous objects, Computer-Aided Design and Applications, vol. 14, No. 1, 2017, pp. 70-82.

[ZCFo4] Zhang XJ, Chen KZ, Feng XA. Optimization of material properties needed for material design of components made of multi-heterogeneous materials. Mater Des 2004;25:369.

[ZCFo5] Feng Zhu, Ke-Zhang Chen, Xin-An Feng, Development of a CAD modeling system for components made of multi heterogeneous materials. Mater Des 2005;26:113.

[ZJRN18] B. Zhang, P. Jaiswal, R. Rai, S. Nelaturi, Additive manufacturing of functionally graded material objects: a review, Journal of Computing and Information Science in Engineering, vol. 18, No. 4, 2018, 041002-16, paper No: JCISE-17-1168.

[ZLLo9] Zhou H, Liu Z, Lu B. Heterogeneous object modeling based on multi-color distance field. Mater Des 2009;30:939.

[ZSo4] Zhang X, Subbarayan G., A constructive approach for heterogeneous material modeling and analysis. Comput Aided Des Appl 2004;1(1-4):171-8.

[ZXYo4] Zhou, M., Xi, J., Yan, J., Modeling and processing of functionally graded materials for rapid prototyping. Journal of Materials Processing Technology 146, 3, 2004. 396402.

[ZT77] Zienkiewicz, O. C., Taylor, R. L., (1977). The finite element method (Vol. 3). London: McGraw-Hill. 